JOURNAL OF POROUS MEDIA

Accepted November $5^{\text {th }} 2019$

ELECTROOSMOTIC FLOW IN A MICROCHANNEL CONTAINING

A POROUS MEDIUM WITH COMPLEX WAVY WALLS

\author{
${ }^{* 1}$ Dharmendra Tripathi, ${ }^{2}$ Shashi Bhushan and ${ }^{3} \mathrm{O}$. Anwar Bég \\ ${ }^{1}$ Department of Science and Humanities, National Institute of Technology, Uttarakhand -246174, India \\ ${ }^{2}$ Department of Mechanical Engineering, Manipal University, Jaipur-303007, India. \\ ${ }^{3}$ Fluid Mechanics, Department of Mechanical and Aeronautical Engineering, Salford University, \\ M54WT, England, UK. \\ *Corresponding author-email: dtripathi@nituk.ac.in
}

\begin{abstract}
In present paper, we simulate the electro-kinetic transport of aqueous solution through a microchannel containing porous media. The micro-channel walls are simulated as complex wavy surface and are modelled by superimposing the three wave functions of different amplitudes but the same wavelength. The micro-channel contains an isotropic, homogenous porous medium, which is analysed with a generalized Darcy law. The nonlinear-coupled governing equations for mass, momentum and electrical potential conservation are simplified using low Reynolds number and long wavelength approximations, and the Debye electro-kinetic linearization. Following non-dimensional transformation of the linearized boundary value problem, closed-form analytical solutions are presented for the velocity components, pressure gradient, local wall shear stress, average flow rate and stream function subject to physically appropriate boundary conditions. Validation with a finite difference method is also conducted. The effect of permeability parameter, Debye length (i.e. characteristic thickness of electrical double layer) and electro-osmotic velocity on flow characteristics is illustrated graphically and interpreted at length. The study finds applications in chromatography, hybrid electro-osmotic micro-pumps, transport phenomena in chemical engineering and energy systems exploiting electro-kinetics.
\end{abstract}

Keywords: Axial electric force; Debye length; Permeability; Helmholtz-Smoluchowski velocity; Porous Medium; Trapping. 


\section{LIST OF SYMBOLS}

\begin{tabular}{|c|c|c|}
\hline Symbols & Description & Unit \\
\hline $\begin{array}{l}\alpha_{1}, \alpha_{2} \\
\alpha_{3}\end{array}$ & Amplitude of waves & $m$ \\
\hline$\delta$ & Wave number & Non-dimensional \\
\hline$\varepsilon$ & Permittivity & $A^{2} s^{4} / \mathrm{kgm}^{2}$ \\
\hline$\lambda$ & Wavelength & $m$ \\
\hline$\lambda_{d}$ & Debye length & $m$ \\
\hline$\mu$ & Fluid viscosity & $\mathrm{kg} / \mathrm{ms}$ \\
\hline $\bar{v}$ & Transverse velocity & $\mathrm{m} / \mathrm{s}$ \\
\hline $\bar{\xi}$ & Axial coordinate & $m$ \\
\hline$\rho$ & Fluid density & $\mathrm{kg} / \mathrm{m}^{3}$ \\
\hline$\tau_{w}$ & Local wall shear stress & $N / m^{2}$ \\
\hline$\phi$ & Electrical potential & $k g m^{2} A^{-1} s^{-3}$ \\
\hline$\psi$ & Stream function & $m^{2} / s$ \\
\hline$\eta$ & Transverse coordinate & $m$ \\
\hline$a$ & Half width of the channel & $m$ \\
\hline$c$ & Average velocity & $\mathrm{m} / \mathrm{s}$ \\
\hline $\bar{k}$ & Permeability & $m^{2}$ \\
\hline$m$ & Electro-osmotic parameter & Non-dimensional \\
\hline$n_{+}$ & Positive ions & Non-dimensional \\
\hline$n_{-}$ & Negative ion & Non-dimensional \\
\hline$n_{0}$ & Number density & $m^{-3}$ \\
\hline $\bar{p}$ & Pressure & $\mathrm{kg} / \mathrm{m} \cdot \mathrm{s}^{2}$ \\
\hline $\bar{t}$ & Time & $s$ \\
\hline $\bar{u}$ & Axial velocity & $\mathrm{m} / \mathrm{s}$ \\
\hline$z_{+}$ & Valency of positive ion & Non-dimensional \\
\hline$z_{-}$ & Valency of negative ion & Non-dimensional \\
\hline$E_{x}$ & Electrical field & $\mathrm{kg} \cdot \mathrm{m} / \mathrm{s}^{3} A$ \\
\hline$L$ & Channel length & $m$ \\
\hline$P e$ & Ionic Peclet number & Non-dimensional \\
\hline$Q$ & Volumetric flow rate & $m^{3} / s$ \\
\hline $\mathrm{Re}$ & Reynolds number & Non-dimensional \\
\hline$S c$ & Schmidt number & Non-dimensional \\
\hline$U_{H S}$ & $\begin{array}{l}\text { Helmholtz-smoluchowski } \\
\text { velocity }\end{array}$ & $\mathrm{m} / \mathrm{s}$ \\
\hline
\end{tabular}




\section{INTRODUCTION}

Flow of fluids in channels under the influence of an electrical field is an important area in both energy sciences and medical engineering. Both electro-hydrodynamics [1] and electro-kinetics (electro-osmotic transport) [2-4] are important aspects of this field. The latter plays a prominent role in micro- and nano-channel systems in ionic flow delivery [5-7], petro-chemical sampling in low-permeability reservoirs [8], geotechnical remediation and field-based stabilization of geological masses [9]. Electrokinetics includes many sophisticated phenomena including streaming currents, streaming potentials, ionic deposition, advection, Joule heating and double layer effects. It has therefore attracted the attention of engineers and scientists in terms of both experimental studies and theoretical investigations. Electro-kinetics can be very successfully utilized to transform mechanical energy into electrical energy, to regulate flow delivery, to achieve specified mass transfer rates etc. Electro-osmotic flows in porous media are also of significance, arise in both biological systems and industrial systems, and have been shown to markedly influence transport rates [10]. The classical approach to simulating transport in porous media is the Darcy model, which is valid for viscous-dominated, low Reynolds number flows. It has been implemented extensively in electro-kinetic modelling for porous media and such studied provide a very important compliment to laboratory-based investigations [11]. Gupta et al. [12] derived approximate mathematical relations for electro-kinetic flow in porous media, valid for general geometries, zeta potentials and electrolyte concentrations and also elongated pores, enabling a robust derivation of the electro-osmotic coefficient. Li et al. [13] studied both experimentally and computationally (with a Lattice Boltzmann method) the electro-osmotic flow (EOF) in micro-porous media, showing that under constant external DC electric field, there is a reduction in flow resistance inside the pores deceases with influx of water into the electro-osmosis pumping section. Gupta et al. [14] analysed the electro-kinetic flow in porous media micro-channels with Stern layer effects, computing expressions for surface charge density, electrical conductivity, and electroosmotic coupling coefficient for various porous structures and physico-chemical boundary conditions. Obliger et al. [15] used a Pore Network Model (PNM) to investigate numerically the influence of pressure, salt concentration, and electric potential gradients on steady-state response of complex charged porous media in a cylindrical channel. Fraia et al. [16] used a finite element method with a characteristic-based split algorithm to investigate the electro-osmotic flow (EOF) in microchannels containing porous media including the influence of electrical charge of solid particles. They solved the combined Laplace, Poisson-Boltzmann and Navier-Stokes equations 
and also considered heat transfer due to electro-osmosis. Tang et al. [17] utilized the representative elementary volume scale porous media model and a lattice Boltzmann algorithm to simulate the combined pressure-driven and electroosmotic flow of Herschel-Bulkley rheological electrolytes in porous media based on the. They evaluated the effects of porosity, solid particle diameter, power law exponent, yield stress and also electric parameters on flow characteristics. She and Liu [18] used a computational finite volume method to simulate the electroosmotic flow in porous media where the solid phase is approximated by cylinders of equal diameters arranged in a regular pattern in response to a given porosity. Brož and Epstein [19] examined experimentally the electrokinetic flow through fine cylindrical capillaries with electrolyte comprising a dilute solution of potassium iodide in purified water. They considered non-Darcy porous media effects and quantified the electroviscous retardation effect for high electrokinetic radius (low double layer thickness). Wu and Keh [20] mathematically studied the steady electrokinetic flow of electrolyte solutions in fibrous porous media, deriving explicit expressions for flow rate, electroosmotic velocity, electric current, effective electric conductivity, and streaming potential as functions of the porosity of the fiber matrix and other electro-kinetic characteristics. Yang et al. [21] have reviewed many applications of electroosmotic pumping in porous media, considering dewatering of toxic sludges and extraction of poisonous heavy metal ions from contaminated soils. Chai et al. [22] have also investigated with a Darcy model, the electro-kinetic dynamics in micro-channels containing variable porosity media.

The above studies have generally considered rigid boundaries for the conduit i.e. microchannel. However, recent progress has been made regarding wavy boundaries and peristaltic propulsion mechanisms in electro-osmotic systems, which can enhance performance and may offer greater efficiencies [23]. Peristalsis is an important biophysical mechanisms arising in many natural systems including reptile locomotion, physiological transport, trans-location of phloem in plants etc. Peristaltic flows involve moving boundary fluid mechanics and are generally analysed with low wavelength and negligible inertial effects. Several mathematical studies of peristaltic hydrodynamics in porous media have been communicated, largely aimed at elucidating impeded flow in the digestive system. El Shehawey et al. [24] used a Newtonian viscous flow model to compute perturbation solutions for stream function and pressure gradient in peristaltic propulsion in a tube containing a Darcian porous medium. Hayat et al. [25] derived analytical solutions for small amplitude ratio in peristaltic hydromagnetic flow of viscoelastic fluids in porous medium with a modified Darcian model. Vajravelu et al. [26] 
computed perturbation solutions for peristalsis pumping and heat transfer in a vertical porous annular region between two concentric tubes. Vasudev et al. [27] computed Darcy number effects in peristaltic flow and heat diffusion in a porous medium channel saturated with Jefferys viscoelastic fluid. Tripathi and Bég [28] who considered rheological peristaltic pumping in porous media conduits using Maxwell's viscoelastic model and a generalized Darcy formulation. Bég et al. [29] used a variational finite element method to investigate species diffusion in pulsating blood flow in non-Darcy porous media, considering different wave forms. Several researchers have also investigated electro-kinetic peristaltic flows. Cho and Chen [30] studied computationally the electroosmotic flow in a microchannel with a complexwavy surface under the influence of a time-varying periodic electric field. They observed that the phases of the electric field and electroosmotic velocity close to the channel wall are almost identical, whereas they differ in the central region of the channel and furthermore that this difference is greater at higher Strouhal number. Al Quddus et al. [31] investigated the influence of surface waviness of walls on electro-kinetic flows in a cylindrical microchannel of finite length, with two reservoirs at the ends, noting that waviness i.e. irregularity in channel wall generates higher concentration and potential gradients across the channel.

In the present investigation, we study the electro-osmotic flow in a finite micro-channel containing a porous medium under wavy surface conditions, which are representative of complex peristaltic propulsion. A Darcy model is employed to mimic bulk impedance effects of the porous medium. We further note that a "wavy surface" is adopted since it is generated by the efficient biological peristalsis propulsion mechanism. The micro-channel is deformable, and so the wavy surface is induced by peristaltic waves propagating along the micro-channel walls. It is not a fixed rigid wavy surface as employed in, for example, solar collectors. This geometry avoids the inherent problems encountered in conventional parallel plate geometries and provides an additional mechanism for regulating transport phenomena in clinical and power-generation systems. Analytical solutions are derived for the transformed, nondimensional linearized boundary value problem. The influence of permeability parameter, Debye length (i.e. characteristic thickness of electrical double layer) and electro-osmotic velocity on pumping characteristics is evaluated in detail. Trapping phenomena are also addressed. The study is relevant to further extending simulations of electro-osmotically driven peristaltic micropumps of interest in medical and energy engineering sciences [32]. 


\section{MATHEMATICAL FORMULATION}

The geometric model for electro-kinetic transport through a finite length ( $L$ ) micro-channel containing a porous medium with complex wavy-wall, as depicted in Fig.1, is modelled following Cho and Chen [30] as:

$$
\bar{h}(\bar{\xi}, \bar{t})=a+\alpha_{1} \sin \frac{2 \pi}{\lambda}(\bar{\xi}-c \bar{t})+\alpha_{2} \sin \frac{4 \pi}{\lambda}(\bar{\xi}-c \bar{t})+\alpha_{3} \sin \frac{8 \pi}{\lambda}(\bar{\xi}-c \bar{t}),
$$

where $a, \alpha_{1}, \alpha_{2}, \alpha_{3}, \lambda, \bar{\xi}, c, \bar{t}, L$ are the half width of the channel, amplitude of three waves, wavelength, axial coordinate, wave velocity, time and channel length. $a, \alpha_{1}, \alpha_{2}$, and $\alpha_{3}$ satisfy the condition $a \geq \sum_{i=1}^{3} \alpha_{i}$.

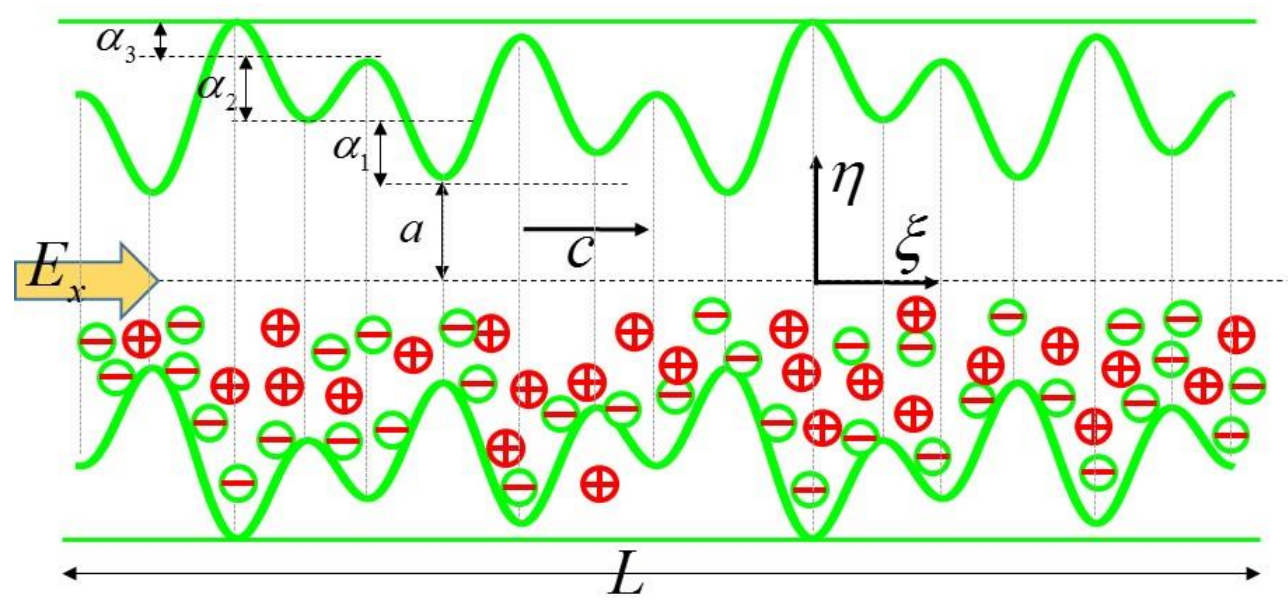

Fig.1. Geometry for electrokinetic transport through complex wavy microchannel containing porous medium.

The porous medium is assumed to be homogenous, isotropic and comprises a matrix permeated by an interconnected network of pores (voids) filled with electrolyte (ionic liquid). Darcy's law is employed which is valid for viscous-dominated flow [33]. High permeability is assumed. An electrical field $\left(E_{x}\right)$ is applied along the longitudinal axis of the micro-channel. Under these assumptions, the governing equations for unsteady, two-dimensional, incompressible flow with an applied electrokinetic body force in the axial (longitudinal) direction through the porous medium micro-channel can be shown to take the form:

$$
\begin{aligned}
& \frac{\partial \bar{u}}{\partial \bar{\xi}}+\frac{\partial \bar{v}}{\partial \bar{\eta}}=0 \\
& \rho\left(\frac{\partial \bar{u}}{\partial \bar{t}}+\bar{u} \frac{\partial \bar{u}}{\partial \bar{\xi}}+\bar{v} \frac{\partial \bar{u}}{\partial \bar{\eta}}\right)=-\frac{\partial \bar{p}}{\partial \bar{\xi}}+\mu\left(\frac{\partial^{2} \bar{u}}{\partial \bar{\xi}^{2}}+\frac{\partial^{2} \bar{u}}{\partial \bar{\eta}^{2}}\right)-\mu \frac{\bar{u}}{\bar{k}}+\rho_{e} \mathrm{E}_{x},
\end{aligned}
$$




$$
\rho\left(\frac{\partial \bar{v}}{\partial \bar{t}}+\bar{u} \frac{\partial \bar{v}}{\partial \bar{\xi}}+\bar{v} \frac{\partial \bar{v}}{\partial \bar{\eta}}\right)=-\frac{\partial \bar{p}}{\partial \bar{\xi}}+\mu\left(\frac{\partial^{2} \bar{v}}{\partial \bar{\xi}^{2}}+\frac{\partial^{2} \bar{v}}{\partial \bar{\eta}^{2}}\right)-\mu \frac{\bar{v}}{\bar{k}}
$$

where $\rho, \bar{u}, \bar{v}, \bar{p}, \mu, \bar{k}$ and $E_{x}$ denote the fluid density, axial velocity, transverse velocity, pressure, fluid viscosity, the permeability of the porous medium and electrokinetic body force. It is important to note that porosity relates to the ratio of volume of the voids to the volume of the solids. It is not required in a Darcy model. The key porous media parameter in our simulation is the permeability $(k)$ which characterizes the hydraulic conductivity of the porous medium and this is the approach adopted. It has been employed by numerous other researchers. We are not studying geometric porosity effects. Our focus is on how the permeability influences transport characteristics. The positive ions $n_{+}$and negative ion $n_{-}$are both assumed to have bulk concentration (number density) $n_{0}$, and a valency of $z_{+}$and $z_{-}$respectively. For simplicity, we consider the electrolyte to be a $z: z$ symmetric electrolyte, i.e. $z_{+}=-z_{-}=z$. The charge number density is related to the electrical potential $(\phi)$ in the transverse direction via the Poisson equation:

$$
\nabla^{2} \phi=-\frac{\rho_{e}}{\varepsilon}
$$

where $\varepsilon$ is the permittivity and $\rho_{e}=e z\left(n^{+}-n^{-}\right)$. Further, in order to determine the potential distribution, charge number density must also be described. For this, the ionic number distributions of the individual species are given by the Nernst-Planck equation for each species as:

$$
\frac{\partial \bar{n}_{ \pm}}{\partial \bar{t}}+\bar{u} \frac{\partial \bar{n}_{ \pm}}{\partial \bar{\xi}}+\bar{v} \frac{\partial \bar{n}_{ \pm}}{\partial \bar{\eta}}=D\left(\frac{\partial^{2} \bar{n}_{ \pm}}{\partial \bar{\xi}^{2}}+\frac{\partial^{2} \bar{n}_{ \pm}}{\partial \bar{\eta}^{2}}\right) \pm \frac{D z e}{k_{B} T}\left(\frac{\partial}{\partial \bar{\xi}}\left(\bar{n}_{ \pm} \frac{\partial \bar{\phi}}{\partial \bar{\xi}}\right)+\frac{\partial}{\partial \bar{\eta}}\left(\bar{n}_{ \pm} \frac{\partial \bar{\phi}}{\partial \bar{\eta}}\right)\right),
$$

where we have assumed equal ionic diffusion coefficients for both the species, and that the mobility of the species is given by the Einstein formula. The following non-dimensional parameters are now introduced to non-dimensionalize the boundary value problem;

$$
\begin{aligned}
& \xi=\frac{\bar{\xi}}{\lambda}, \eta=\frac{\bar{\eta}}{a}, t=\frac{c \bar{t}}{\lambda}, u=\frac{\bar{u}}{c}, v=\frac{\bar{v}}{c \delta}, \delta=\frac{a}{\lambda}, \\
& h=\frac{\bar{h}}{a}=1+\varphi_{1} \sin 2 \pi(\xi-t)+\varphi_{2} \sin 4 \pi(\xi-t)+\varphi_{3} \sin 8 \pi(\xi-t), \\
& \varphi_{1}=\frac{\alpha_{1}}{a}, \varphi_{2}=\frac{\alpha_{2}}{a}, \varphi_{3}=\frac{\alpha_{3}}{a}, p=\frac{\bar{p} a^{2}}{\mu c \lambda}, \operatorname{Re}=\frac{\rho c a}{\mu}, \mathrm{k}=\frac{\bar{k}}{a^{2}}, \phi=\frac{\bar{\phi}}{\zeta}, n=\frac{\bar{n}}{n_{0}},
\end{aligned}
$$


where $\delta$ is wave number, $\operatorname{Re}$ is the Reynolds number, $\zeta$ is the zeta potential, and the nonlinear terms in the Nernst Planck equations are $O\left(P e \delta^{2}\right)$, where $P e=\operatorname{ReSc}$ represents the ionic Peclet number and $S c=\mu / \rho D$ denotes the Schmidt number. Therefore, the nonlinear terms may be dropped in the limit that $R e, P e, \delta<1$. In this limit, Poisson equation is obtained as:

$$
\frac{\partial^{2} \phi}{\partial \eta^{2}}=-m^{2}\left(\frac{n_{+}-n_{-}}{2}\right),
$$

where $m=a e z \sqrt{\frac{2 n_{0}}{\varepsilon K_{B} T}}=\frac{a}{\lambda_{d}}$, is known as the electro-osmotic parameter and $\lambda_{d} \propto \frac{1}{m}$ is Debye length or characteristic thickness of electrical double layer (EDL).

The ionic distribution may be determined by means of the simplified Nernst Planck equations:

$$
0=\frac{\partial^{2} n_{ \pm}}{\partial \eta^{2}} \pm \frac{\partial}{\partial \eta}\left(n_{ \pm} \frac{\partial \phi}{\partial \eta}\right)
$$

subjected to $n_{ \pm}=1$ at $\phi=0$ and $\partial n_{ \pm} / \partial \eta=0$ where $\partial \phi / \partial \eta=0$ (bulk conditions). These yield the much celebrated Boltzmann distribution for the ions:

$$
n_{ \pm}=e^{\mp \phi}
$$

Combining equations (8) and (10), we obtain the Poisson-Boltzmann paradigm for the potential determining the potential distribution:

$$
\frac{\partial^{2} \phi}{\partial \eta^{2}}=m^{2} \sinh (\phi) .
$$

In order to make further analytical progress, we must simplify equation (11). Equation (11) may be linearized under the low-zeta potential approximation. This assumption is not ad hoc since for a wide range of $\mathrm{pH}$, the magnitude of zeta potential is less than $25 \mathrm{mV}$. Therefore, equation (11) reduces to:

$$
\frac{\partial^{2} \phi}{\partial \eta^{2}}=m^{2} \phi
$$

which may be solved subjected to $\left.\frac{\partial \phi}{\partial \eta}\right|_{\eta=0}=0$ and $\left.\phi\right|_{\eta=h}=1$, the potential function is obtained as: 


$$
\phi=\frac{\cosh (m \eta)}{\cosh (m h)}
$$

Under the assumptions of long wave length and low Reynolds number, the governing conservation equations reduce to:

$$
\begin{aligned}
& \frac{\partial u}{\partial \xi}+\frac{\partial v}{\partial \eta}=0 \\
& \frac{\partial p}{\partial \xi}=\frac{\partial^{2} u}{\partial \eta^{2}}-\frac{u}{k}+m^{2} U_{H S} \phi \\
& \frac{\partial p}{\partial \eta}=0
\end{aligned}
$$

where $U_{H S}=-\frac{E_{x} \varepsilon \zeta}{\mu c}$ is the Helmholtz-Smoluchowski velocity or maximum electro-osmotic velocity. The imposed boundary conditions are:

$$
\left.\frac{\partial u}{\partial \eta}\right|_{\eta=0}=0,\left.u\right|_{\eta=h}=0,\left.v\right|_{\eta=0}=0,\left.v\right|_{\eta=h}=\frac{\partial h}{\partial t},\left.p\right|_{\xi=0}=p_{0} \text { and }\left.p\right|_{\xi=L}=p_{L}
$$

Integrating Eq. (15) and using boundary conditions (17), the axial velocity is obtained as:

$$
u=k \frac{\partial p}{\partial \xi}\left\{\frac{\cosh \left(\frac{\eta}{\sqrt{k}}\right)}{\cosh \left(\frac{h}{\sqrt{k}}\right)}-1\right\}+\frac{m^{2} U_{H S}}{m^{2}-\frac{1}{k}}\left\{\frac{\cosh \left(\frac{\eta}{\sqrt{k}}\right)}{\cosh \left(\frac{h}{\sqrt{k}}\right)}-\frac{\cosh (m \eta)}{\cosh (m h)}\right\}
$$

Using Eq. (18) and boundary condition (17), the transverse velocity from the continuity equation (14) is obtained as:

$$
\begin{aligned}
v=- & k\left\{\frac{\partial^{2} p}{\partial \xi^{2}}\left(\frac{\sqrt{k} \sinh \left(\frac{\eta}{\sqrt{k}}\right)}{\cosh \left(\frac{h}{\sqrt{k}}\right)}-\eta\right)+\frac{\partial p}{\partial \xi} \frac{\partial h}{\partial \xi} \frac{\sinh \left(\frac{\eta}{\sqrt{k}}\right) \tanh \left(\frac{h}{\sqrt{k}}\right)}{\cosh \left(\frac{h}{\sqrt{k}}\right)}\right\}+ \\
& \frac{m^{2} U_{H S}}{m^{2}-\frac{1}{k}}\left\{\frac{\sinh \left(\frac{\eta}{\sqrt{k}}\right) \tanh \left(\frac{h}{\sqrt{k}}\right)}{\cosh \left(\frac{h}{\sqrt{k}}\right)}-\frac{\sinh (m \eta) \tanh (m h)}{\cosh (m h)}\right\} \frac{\partial h}{\partial \xi}
\end{aligned}
$$

Furthermore, utilizing Eq. (19) and boundary condition (17), the pressure gradient emerges as: 
$\frac{\partial p}{\partial \xi}=\frac{1}{k^{3 / 2}\left(\frac{h}{\sqrt{k}}-\tanh \left(\frac{h}{\sqrt{k}}\right)\right)}\left[G_{0}(t)+\int \frac{\partial h}{\partial t} d \xi-\frac{m^{2} U_{H S}}{m^{2}-\frac{1}{k}}\left\{\sqrt{k} \tanh \left(\frac{h}{\sqrt{k}}\right)-\frac{\tanh (m h)}{m}\right\}\right]$

where $G_{0}(t)$ is arbitrary function of time ( $t$ ) to be evaluated by using finite length boundary conditions. The pressure difference can be computed along the axial length by the expression:

$$
\Delta p=p(\xi, t)-p(0, t)=\int_{0}^{\xi} \frac{\partial p}{\partial s} d s
$$

Here $G_{0}(t)$ is expressed as:

$$
G_{0}(t)=\frac{\left.\left(p_{l}-p_{0}\right)-\int_{0}^{L} \frac{1}{k^{3 / 2}\left(\frac{h}{\sqrt{k}}-\tanh \left(\frac{h}{\sqrt{k}}\right)\right.}\right)\left[\int \frac{\partial h}{\partial t} d \xi-\frac{m^{2} U_{H S}}{m^{2}-\frac{1}{k}}\left\{\sqrt{k} \tanh \left(\frac{h}{\sqrt{k}}\right)-\frac{\tanh (m h)}{m}\right\}\right] d \xi}{\int_{0}^{L} \frac{1}{k^{3 / 2}\left(\frac{h}{\sqrt{k}}-\tanh \left(\frac{h}{\sqrt{k}}\right)\right)} d \xi} .
$$

The local wall shear stress is defined as:

$\tau_{w}=\left.\frac{\partial u}{\partial \eta}\right|_{\eta=h}=\sqrt{k} \tanh \left(\frac{h}{\sqrt{k}}\right) \frac{\partial p}{\partial \xi}-\frac{m^{2} U_{H S}}{m^{2}-\frac{1}{k}}\left\{\frac{\tanh \left(\frac{h}{\sqrt{k}}\right)}{\sqrt{k}}-m \tanh (m h)\right\}$.

The volumetric flow rate is defined as:

$$
Q(\xi, t)=\int_{0}^{h} u d \eta=k^{3 / 2} \frac{\partial p}{\partial \xi}\left\{\tanh \left(\frac{h}{\sqrt{k}}\right)-\frac{h}{\sqrt{k}}\right\}+\frac{m^{2} U_{H S}}{m^{2}-\frac{1}{k}}\left\{\sqrt{k} \tanh \left(\frac{h}{\sqrt{k}}\right)-\frac{\tanh (m h)}{m}\right\} .
$$

The transformations between a wave frame $(x, y)$ moving with velocity $c$ and the fixed frame ( $\xi, \eta)$ are given by :

$$
\xi=x-c t, \quad \eta=y, \quad u=u_{w}+c, \quad v=v_{w}
$$

where $\left(u_{w}, v_{w}\right)$ and $(u, v)$ are the velocity components in the wave and fixed frame respectively. 
The volumetric flow rate in the wave frame is given by

$$
q_{w}=\int_{0}^{h} u_{w} d y=\int_{0}^{h}(u-1) d y,
$$

which, on integration, yields:

$$
q_{w}=Q-h
$$

Averaging volumetric flow rate along one time period, we get:

$$
\bar{Q}=\int_{0}^{1} Q d t=\int_{0}^{1}\left(q_{w}+h\right) d t,
$$

which, on integration, yields

$$
\bar{Q}=q_{w}+1=Q+1-h
$$

Using Eqs.(18 \& 19), the stream function in the wave frame (obeying the Cauchy-Riemann equations, $u_{w}=\frac{\partial \psi}{\partial y}$ and $\left.v_{w}=-\frac{\partial \psi}{\partial x}\right)$ takes the form:

$\psi=k \frac{\partial p}{\partial x}\left\{\frac{\sqrt{k} \sinh \left(\frac{y}{\sqrt{k}}\right)}{\cosh \left(\frac{h}{\sqrt{k}}\right)}-y\right\}+\frac{m^{2} U_{H S}}{m^{2}-\frac{1}{k}}\left\{\frac{\sqrt{k} \sinh \left(\frac{y}{\sqrt{k}}\right)}{\cosh \left(\frac{h}{\sqrt{k}}\right)}-\frac{\sinh (m y)}{m \cosh (m h)}\right\}$.

All the above expressions will reduce to the corresponding expressions for viscous flow through finite length porous channel with $U_{H S}=0$ i.e. for vanishing Helmholtz-Smoluchowski velocity (negligible electrokinetic effect). It is also noteworthy that the expressions (18)-(30) contract to the case for electro-kinetic peristaltic transport through a very thin electric double layer with electro-osmotic parameter $m \rightarrow \infty$. Finally for $k \rightarrow \infty$, permeability becomes infinite and the general model reduces to purely electro-kinetic transport in electrolytic fluid media without porous medium drag forces.

\section{VALIDATION WITH KELLER BOX FINITE DIFFERENCE METHOD}

To verify the present analytical solutions, an implicit finite difference method originated by Keller [34] has also been employed to solve the boundary value problem defined by eqns. (14)- 
(16) under conditions (17). Recent electromagnetic linear and nonlinear problems which have employed Keller's method include magnetized extending cylinder flows [35], Hall magnetogas dynamic generator slip flows [36] and radiative-convective Casson slip boundary layer flows [37]. Keller's method provides unconditional stability and rapid convergence for strongly non-linear flows. It involves four key stages, summarized below.

1) Reduction of the $N^{\text {th }}$ order partial differential equation system to $N$ first order equations

2) Finite difference discretization of reduced equations

3) Quasilinearization of non-linear Keller algebraic equations

4) Block-tridiagonal elimination of linearized Keller algebraic equations

A two-dimensional computational grid (mesh) which is suitable for the micro-channel domain is imposed on the $\xi-\eta$ plane as sketched in Fig.2. The stepping process is defined by:

$$
\begin{array}{ll}
\eta_{0}=0, & \eta_{j}=\eta_{j-1}+h_{j}, \quad j=1,2, \ldots, J, \quad \eta_{J} \equiv \eta_{\infty} \\
\xi^{0}=0, & \xi^{n}=\xi^{n-1}+k_{n}, \quad n=1,2, \ldots, N
\end{array}
$$

where $k_{n}$ and $h_{j}$ denote the step distances in the $\xi$ (streamwise) and $\eta$ (spanwise) directions respectively.

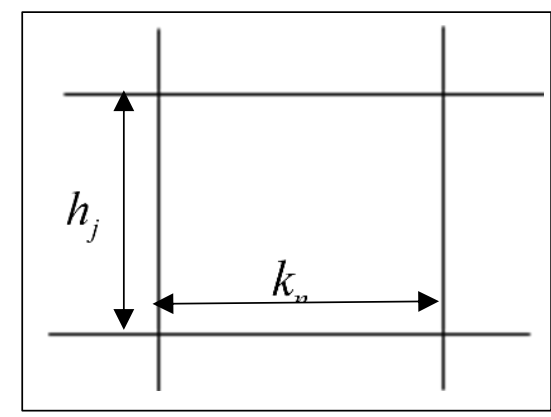

Fig.2. Keller Box element.

If $g_{j}^{n}$ denotes the value of a general variable e.g. $u, v, p, \phi$, at $\left(\eta_{j}, \xi^{n}\right)$, then the variables and derivatives of Eqns. (14) - (16) at $\left(\eta_{j-1 / 2}, \xi^{n-1 / 2}\right)$ are replaced by: 


$$
\begin{gathered}
g_{j-1 / 2}^{n-1 / 2}=\frac{1}{4}\left(g_{j}^{n}+g_{j-1}^{n}+g_{j}^{n-1}+g_{j-1}^{n-1}\right), \\
\left(\frac{\partial g}{\partial \eta}\right)_{j-1 / 2}^{n-1 / 2}=\frac{1}{2 h_{j}}\left(g_{j}^{n}-g_{j-1}^{n}+g_{j}^{n-1}-g_{j-1}^{n-1}\right), \\
\left(\frac{\partial g}{\partial \xi}\right)_{j-1 / 2}^{n-1 / 2}=\frac{1}{2 k^{n}}\left(g_{j}^{n}-g_{j-1}^{n}+g_{j}^{n-1}-g_{j-1}^{n-1}\right),
\end{gathered}
$$

The finite-difference approximation of equations (14) - (16) for the mid-point $\left(\eta_{j-1 / 2}, \xi^{n}\right)$ are readily obtained. Details are excluded here for brevity. The complete system is formulated as a block matrix system, where each element in the coefficient matrix is a matrix itself. Then, this system is solved using the efficient Keller-box method. The numerical results are affected by the number of mesh points in both directions. After some trials in the $\eta$-direction (transverse coordinate) a larger number of mesh points are selected whereas in the $\xi$ direction (axial coordinate) significantly less mesh points are utilized. $\eta_{\max }$ has been set at $\pm 1.0 . \xi_{\max }$ is set at 2.0 for this flow domain. Mesh independence testing is also performed to ensure that the converged solutions are correct. The computer program of the algorithm is executed in MATLAB running on a PC. The function $G_{0}(t)$ is arbitrary function of time $(t)$ and is again computed using finite length boundary conditions in the Keller box program. Comparison solutions for the analytical and Keller box method (KBM) solutions are provided in Tables 13 for pressure difference values with different $m, U_{H S}$ and $k$ values.

Table 1: Analytical and Keller box solutions for pressure difference $(\Delta p)$ versus axial displacement $(\xi)$ at $\varphi_{1}=0.1, \varphi_{2}=0.2, \varphi_{3}=0.3$ for different electro-osmotic parameters $(\mathrm{m})$.

\begin{tabular}{|l|c|l|c|l|c|l|}
\hline \multirow{2}{*}{$\xi$} & \multicolumn{6}{c|}{$\Delta p$ for different $m$ at $U_{H S}=1, k=0.1$} \\
\hline & Analytical & KBM & Analytical & KBM & Analytical & KBM \\
\hline \multirow{2}{*}{$m=2$} & \multicolumn{2}{|c|}{$m=4$} & \multicolumn{2}{c|}{$m \rightarrow \infty$} \\
\hline 0 & 0 & 0 & 0 & 0 & 0 & 0 \\
\hline 0.25 & 0.9407 & 0.9409 & 0.9231 & 0.9241 & 0.9007 & 0.9010 \\
\hline 0.5 & 0.0987 & 0.0981 & 0.0999 & 0.0994 & 0.0915 & 0.0921 \\
\hline 0.75 & 0.3212 & 0.3220 & 0.3152 & 0.3148 & 0.3048 & 0.3051 \\
\hline 1.0 & -0.0025 & -0.0031 & -0.0028 & -0.0024 & -0.0027 & -0.0030 \\
\hline 1.25 & 0.9546 & 0.9493 & 0.9381 & 0.9377 & 0.915 & 0.919 \\
\hline
\end{tabular}




\begin{tabular}{|l|c|l|c|l|c|l|}
\hline 1.5 & 0.08346 & 0.08351 & 0.0837 & 0.0840 & 0.07595 & 0.07598 \\
\hline 1.75 & 0.2475 & 0.2481 & 0.2392 & 0.2388 & 0.2328 & 0.2329 \\
\hline 2.0 & 0 & 0 & 0 & 0 & 0 & 0 \\
\hline
\end{tabular}

Table 2: Analytical and Keller box solutions for pressure difference $(\Delta p)$ versus axial displacement $(\xi)$ at $\varphi_{1}=0.1, \varphi_{2}=0.2, \varphi_{3}=0.3$ for different $\mathrm{H}-\mathrm{S}$ velocity parameters $\left(U_{H S}\right)$.

\begin{tabular}{|l|c|l|c|l|c|l|}
\hline \multirow{2}{*}{$\xi$} & \multicolumn{6}{|c|}{$\Delta p$ for different $U_{H S}$ at $k=0.1, m=2$} \\
\hline & Analytical & KBM & Analytical & KBM & Analytical & KBM \\
\hline & \multicolumn{2}{|c|}{$U_{H S}=-1$} & \multicolumn{2}{|c|}{$U_{H S}=0$} & \multicolumn{2}{|c|}{$U_{H S}=+1$} \\
\hline 0 & 0 & 0 & 0 & 0 & 0 & 0 \\
\hline 0.25 & 0.7977 & 0.8001 & 0.8692 & 0.8696 & 0.9407 & 0.9411 \\
\hline 0.5 & 0.0558 & 0.0554 & 0.0773 & 0.0775 & 0.0987 & 0.0989 \\
\hline 0.75 & 0.2596 & 0.2592 & 0.2904 & 0.2910 & 0.3212 & 0.3215 \\
\hline 1.0 & -0.00195 & -0.00191 & -0.0022 & -0.0023 & -0.0025 & -0.0027 \\
\hline 1.25 & 0.8081 & 0.8079 & 0.8813 & 0.8816 & 0.9546 & 0.9548 \\
\hline 1.5 & 0.04455 & 0.04452 & 0.064 & 0.061 & 0.08346 & 0.08349 \\
\hline 1.75 & 0.20566 & 0.20559 & 0.2266 & 0.2268 & 0.2475 & 0.2472 \\
\hline 2.0 & 0 & 0 & 0 & 0 & 0 & 0 \\
\hline
\end{tabular}

Table 3: Analytical and Keller box solutions for pressure difference $(\Delta p)$ versus axial displacement $(\xi)$ at $\varphi_{1}=0.1, \varphi_{2}=0.2, \varphi_{3}=0.3$ for different permeability parameters $(k)$.

\begin{tabular}{|l|c|l|c|l|l|l|}
\hline \multicolumn{1}{|c|}{$\xi$} & \multicolumn{6}{c|}{$\Delta p$ for different $k$ at $m=2, U_{H S}=1$} \\
\hline & Analytical & KBM & Analytical & KBM & Analytical & KBM \\
\hline & \multicolumn{2}{|c|}{$k=0.1$} & \multicolumn{2}{|c|}{$k=0.2$} & \multicolumn{2}{|c|}{$k=0.3$} \\
\hline 0 & 0 & 0 & 0 & 0 & 0 & 0 \\
\hline 0.25 & 0.9407 & 0.9409 & 0.6811 & 0.68153 & 0.5984 & 0.5981 \\
\hline 0.5 & 0.0987 & 0.0984 & 0.1136 & 0.11421 & 0.1203 & 0.1205 \\
\hline 0.75 & 0.3212 & 0.3214 & 0.2504 & 0.25061 & 0.2287 & 0.2289 \\
\hline 1.0 & -0.0025 & -0.0026 & -0.0025 & -0.0027 & -0.0025 & -0.0028 \\
\hline 1.25 & 0.9546 & 0.9549 & 0.6947 & 0.69490 & 0.612 & 0.61311 \\
\hline
\end{tabular}




\begin{tabular}{|l|c|l|c|l|c|l|}
\hline 1.5 & 0.08346 & 0.08347 & 0.09885 & 0.09886 & 0.1055 & 0.10547 \\
\hline 1.75 & 0.2475 & 0.2477 & 0.18144 & 0.18439 & 0.1606 & 0.16058 \\
\hline 2.0 & 0 & 0 & 0 & 0 & 0 & 0 \\
\hline
\end{tabular}

Generally very good agreement has been achieved between the closed-form and Keller box computations. Confidence in the present analytical solutions, which are used to plot all graphs, is therefore high.

\section{RESULTS AND DISCUSSION}

Graphical solutions have been presented in figs. 3-9. The peristaltic electro-kinetic porous media boundary value problem is studied for the influence primarily of three hydrodynamic parameters - maximum electro-osmotic velocity $\left(U_{H S}\right)$, electro-osmotic parameter $(m)$ and the porous medium permeability parameter $(k)$ - and also time $(t)$. In all plots default values for other parameters are set as $\varphi_{1}=0.1, \varphi_{2}=0.2, \varphi_{3}=0.3, x=1.0, t=0, \frac{\partial p}{\partial x}=1$.

(a)

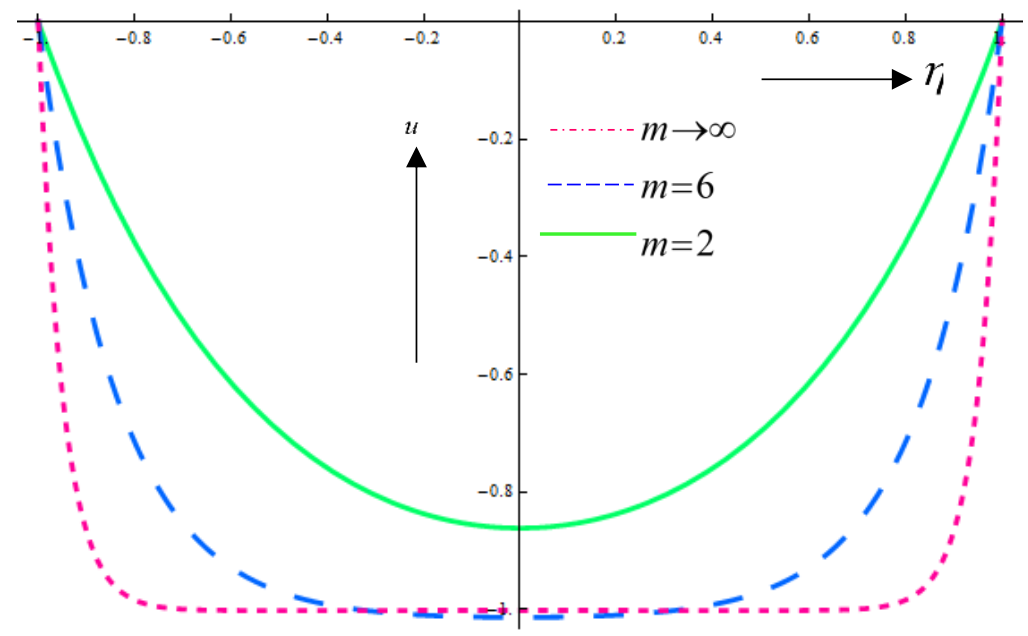


(b)

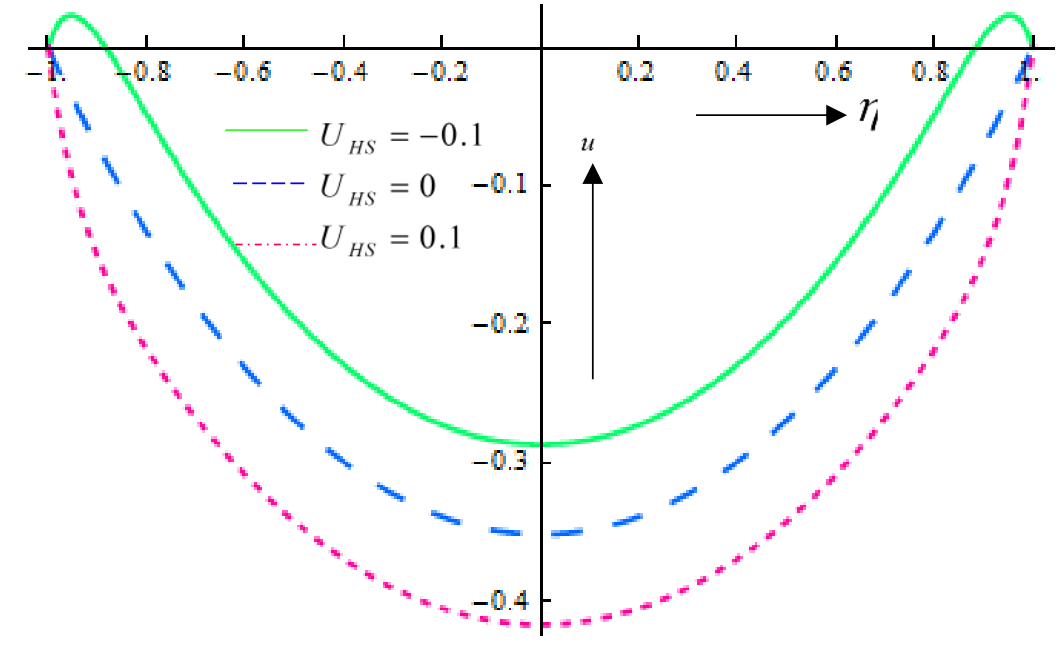

(c)

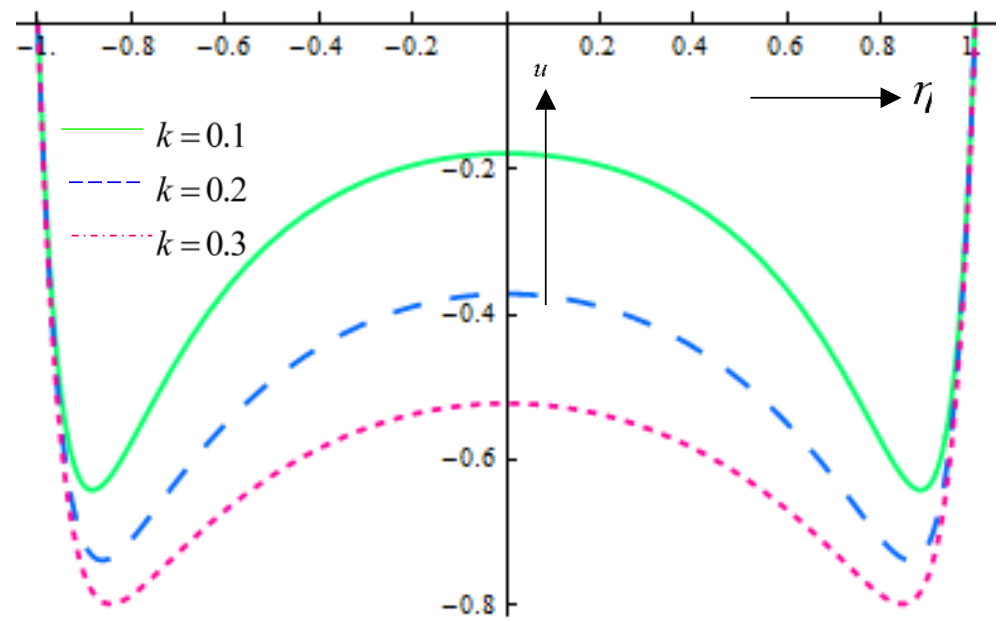

Fig.3. Velocity profile (axial velocity vs. transverse coordinate at $\varphi_{1}=0.1, \varphi_{2}=0.2, \varphi_{3}=0.3, x=1.0, t=0, \frac{\partial p}{\partial x}=1$ and (a) $U_{H S}=1, k=1$ (b) $m=20, k=1$ (c) $U_{H S}=1, m=20$.

(a) $t=0.2$

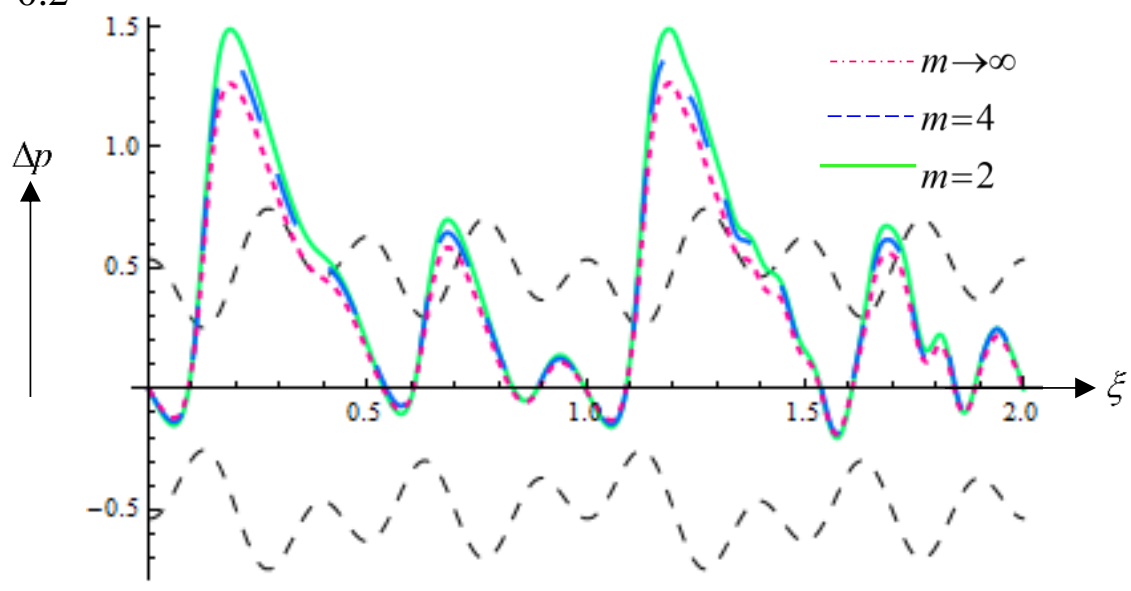

(b) $t=0.6$

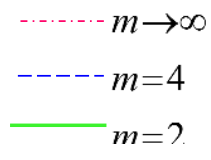




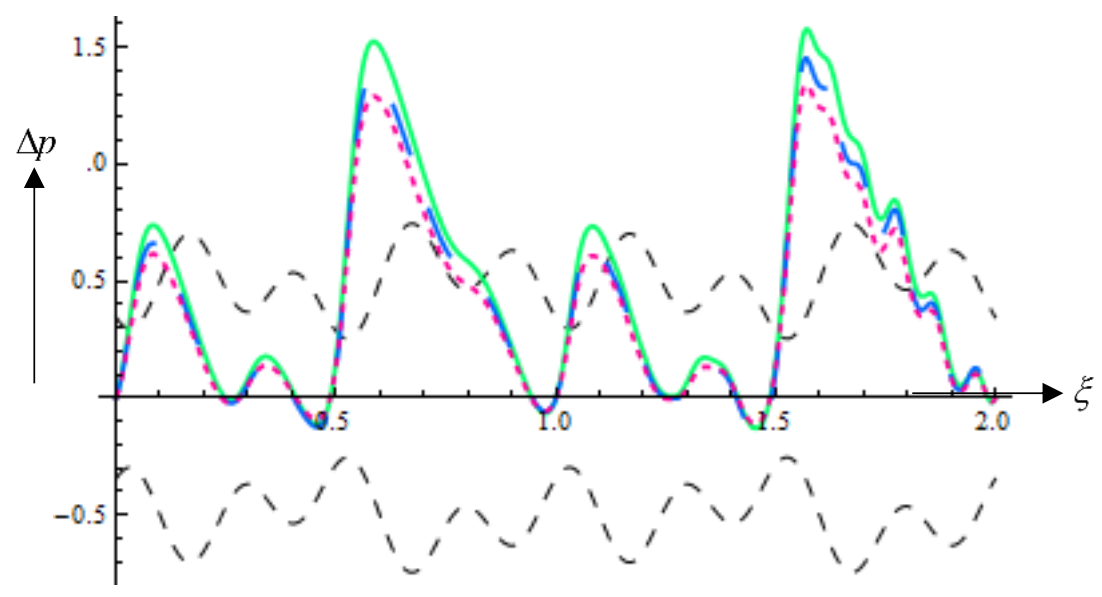

Fig.4. Pressure distribution along the length of channel at $\varphi_{1}=0.1, \varphi_{2}=0.2, \varphi_{3}=0.3$, $l=2, p_{l}=p_{0}=0, U_{H S}=5, k=0.1$. Color lines represent the pressure distribution and Black dotted lines show the complex wave propagation.

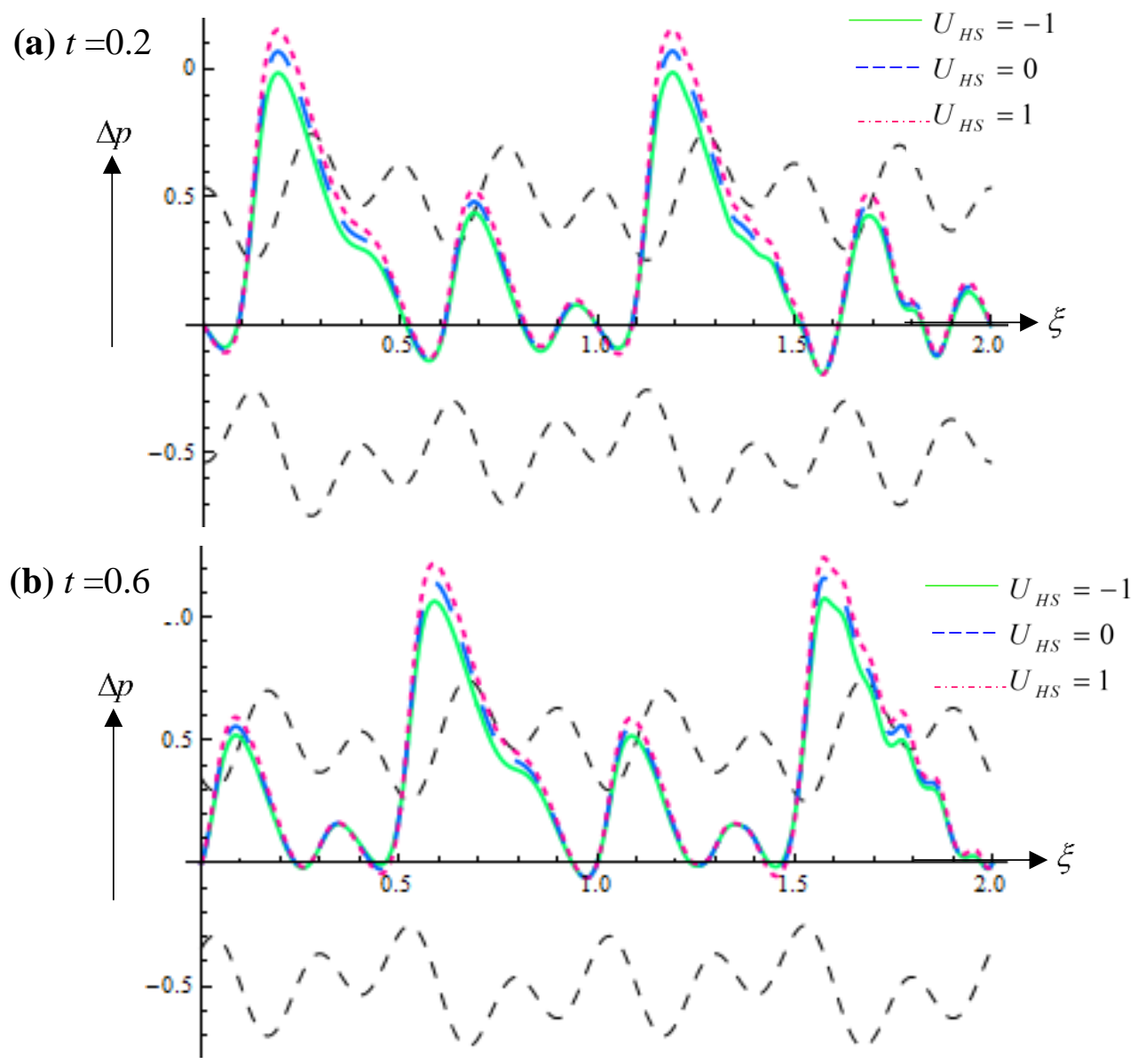

Fig.5. Pressure distribution along the length of channel at $\varphi_{1}=0.1, \varphi_{2}=0.2, \varphi_{3}=0.3$, $l=2, p_{l}=p_{0}=0, m=2, k=0.1$. Color lines represent the pressure distribution and Black dotted lines show the complex wave propagation. 


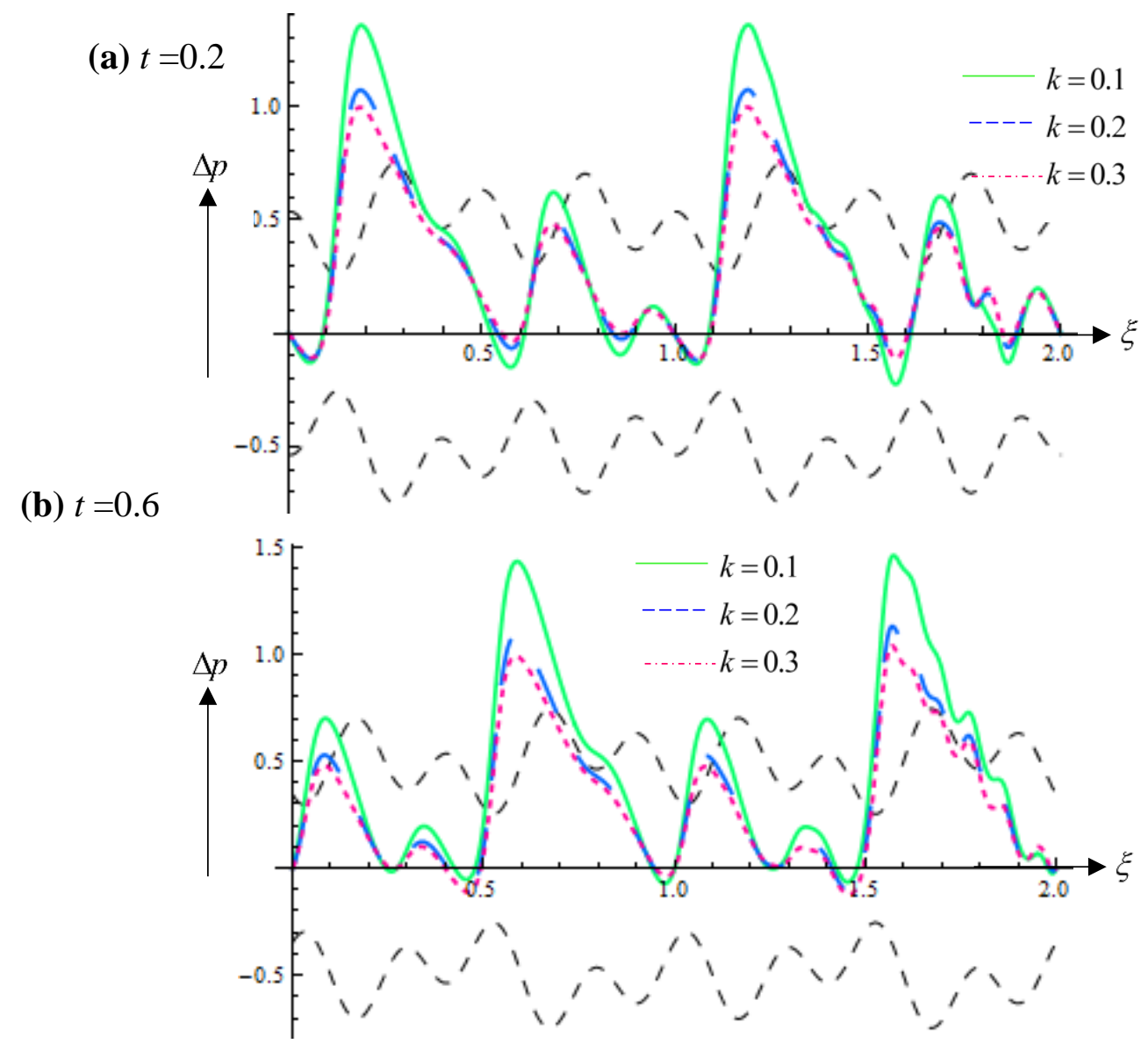

Fig.6. Pressure distribution along the length of channel at $\varphi_{1}=0.1, \varphi_{2}=0.2, \varphi_{3}=0.3$, $l=2, p_{l}=p_{0}=0, U_{H S}=5, m=1$. Color lines represent the pressure distribution and Black dotted lines show the complex wave propagation.

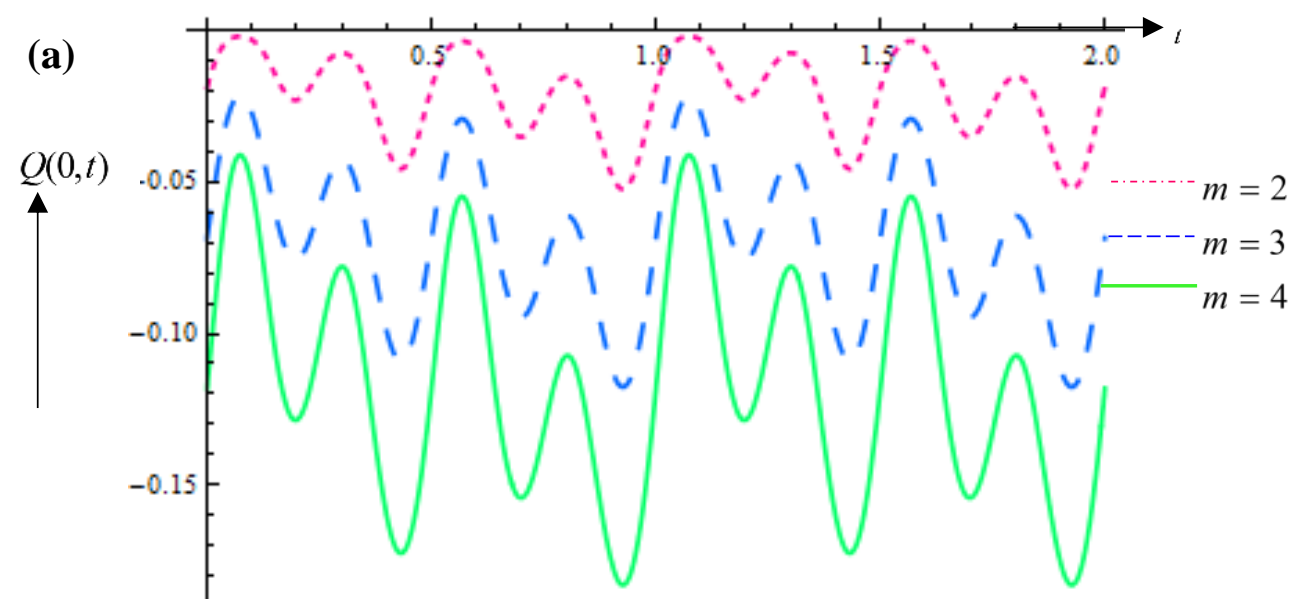

(b) 

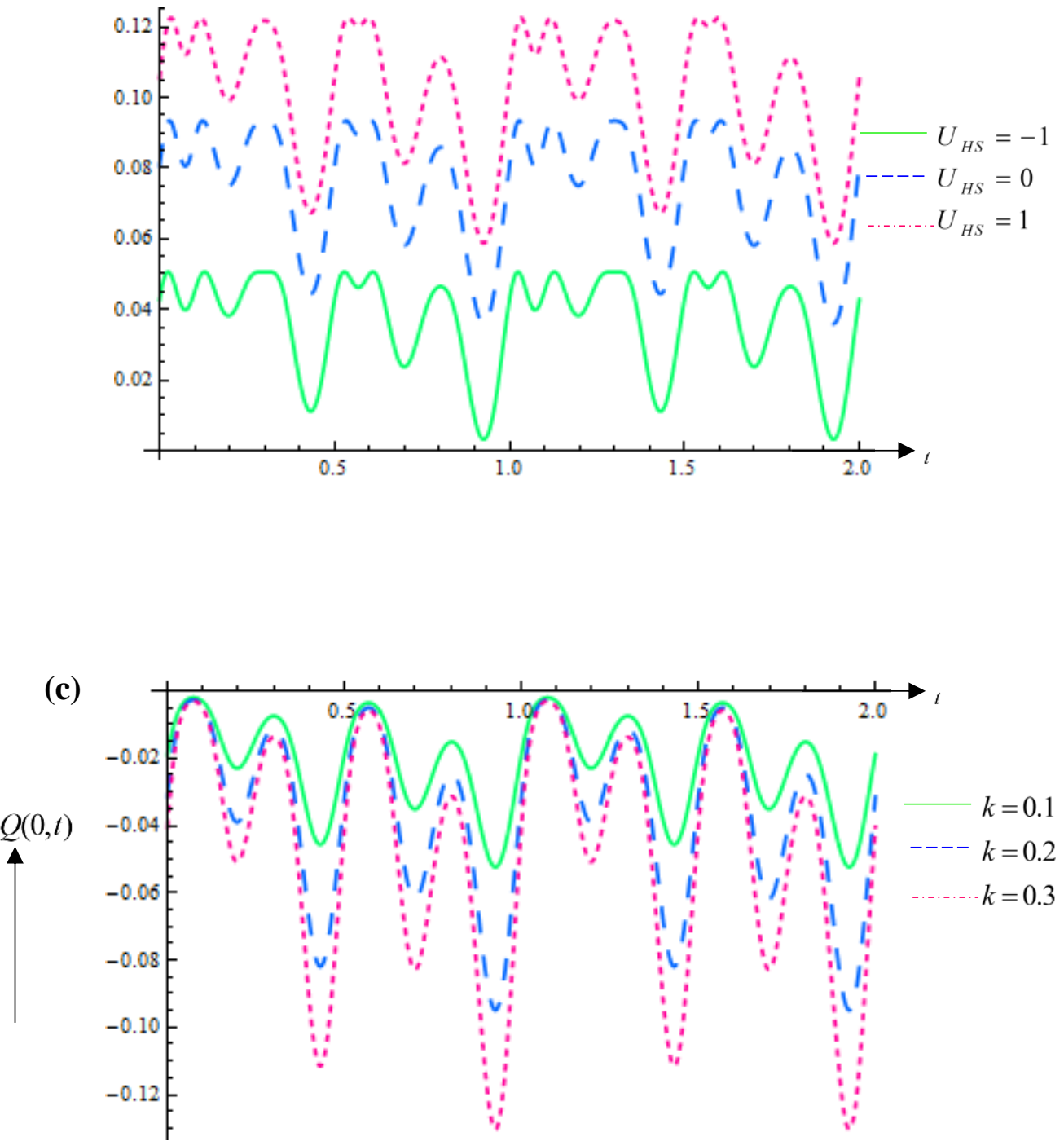

Fig.7. Initial flow rate against the time at $\varphi_{1}=0.1, \varphi_{2}=0.2, \varphi_{3}=0.3, \frac{\partial p}{\partial x}=1$ and (a) $U_{H S}=1, k=0.1$ (b) $m=1, k=0.1$ (c) $U_{H S}=1, m=1$.

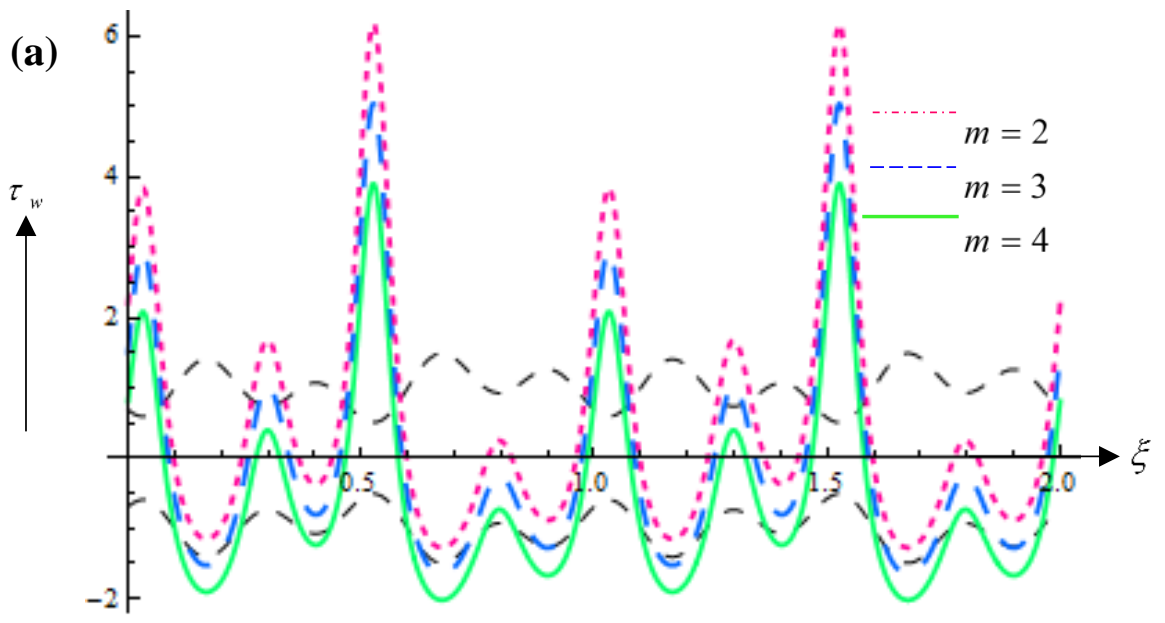




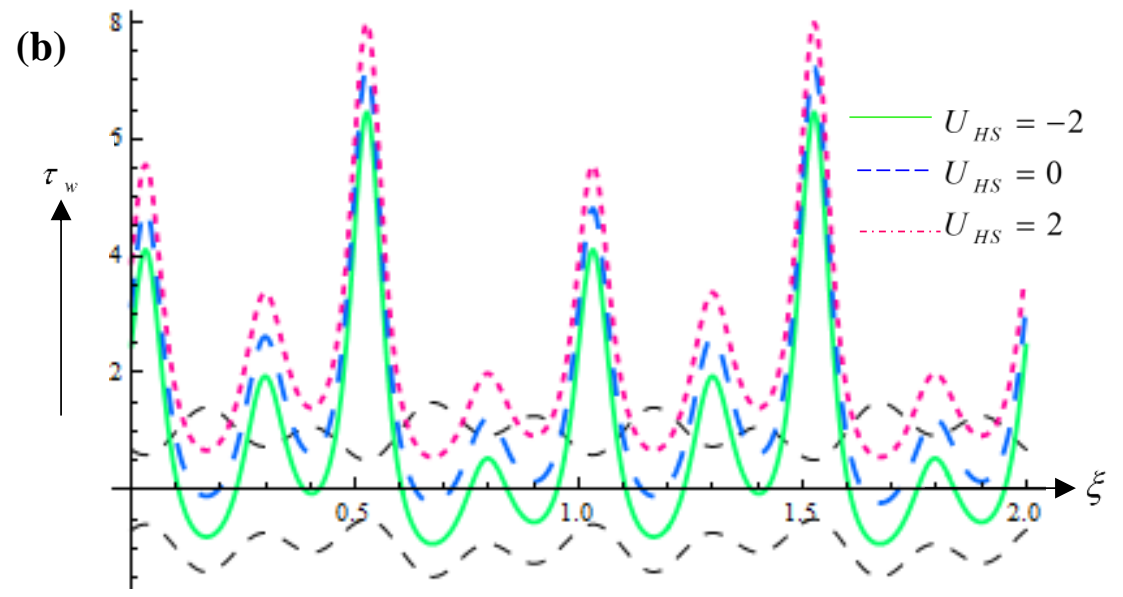

(c)

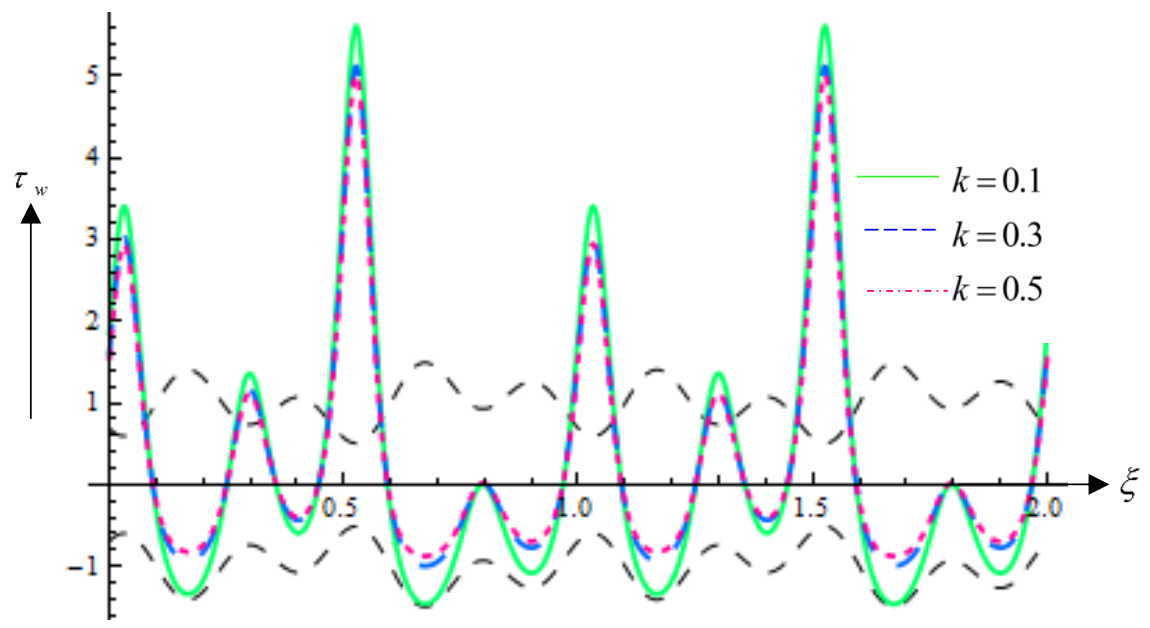

Fig.8. Local wall shear stress along the length of channel at $\varphi_{1}=0.1, \varphi_{2}=0.2, \varphi_{3}=0.3$, $l=2, p_{l}=p_{0}=0, t=0.6$, for (a) $U_{H S}=1, k=0.1$ (b) $m=1, k=0.1$ (c) $U_{H S}=1, m=1$. Color lines represent the Local wall shear stress and Black dotted lines show the train complex wave propagation. 
(a)

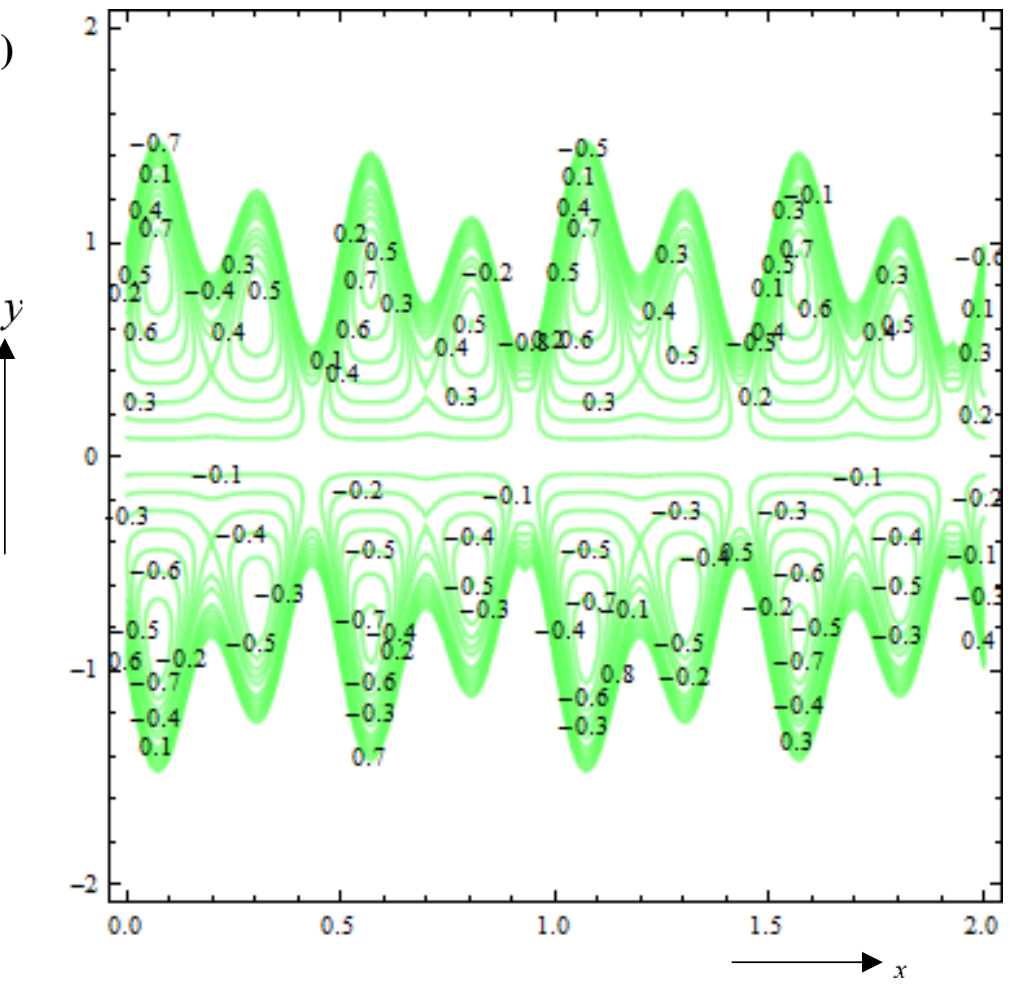

(b)

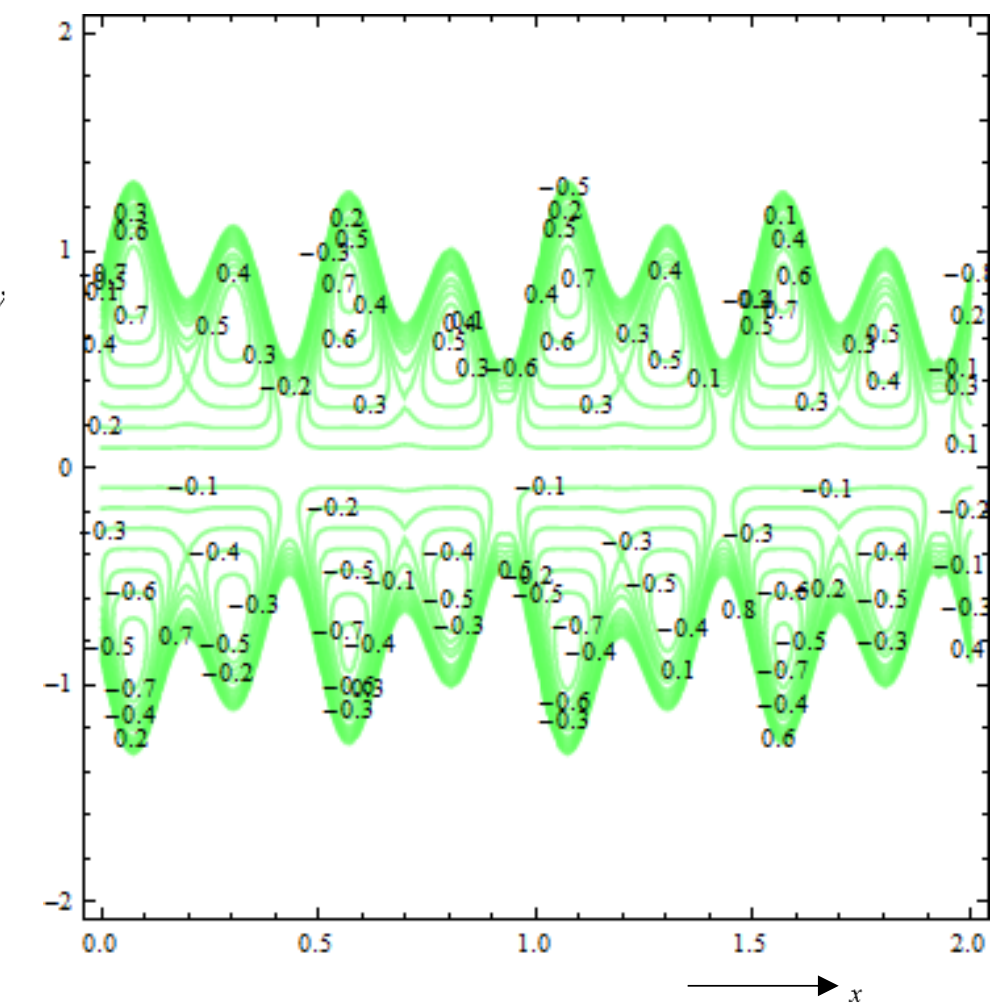



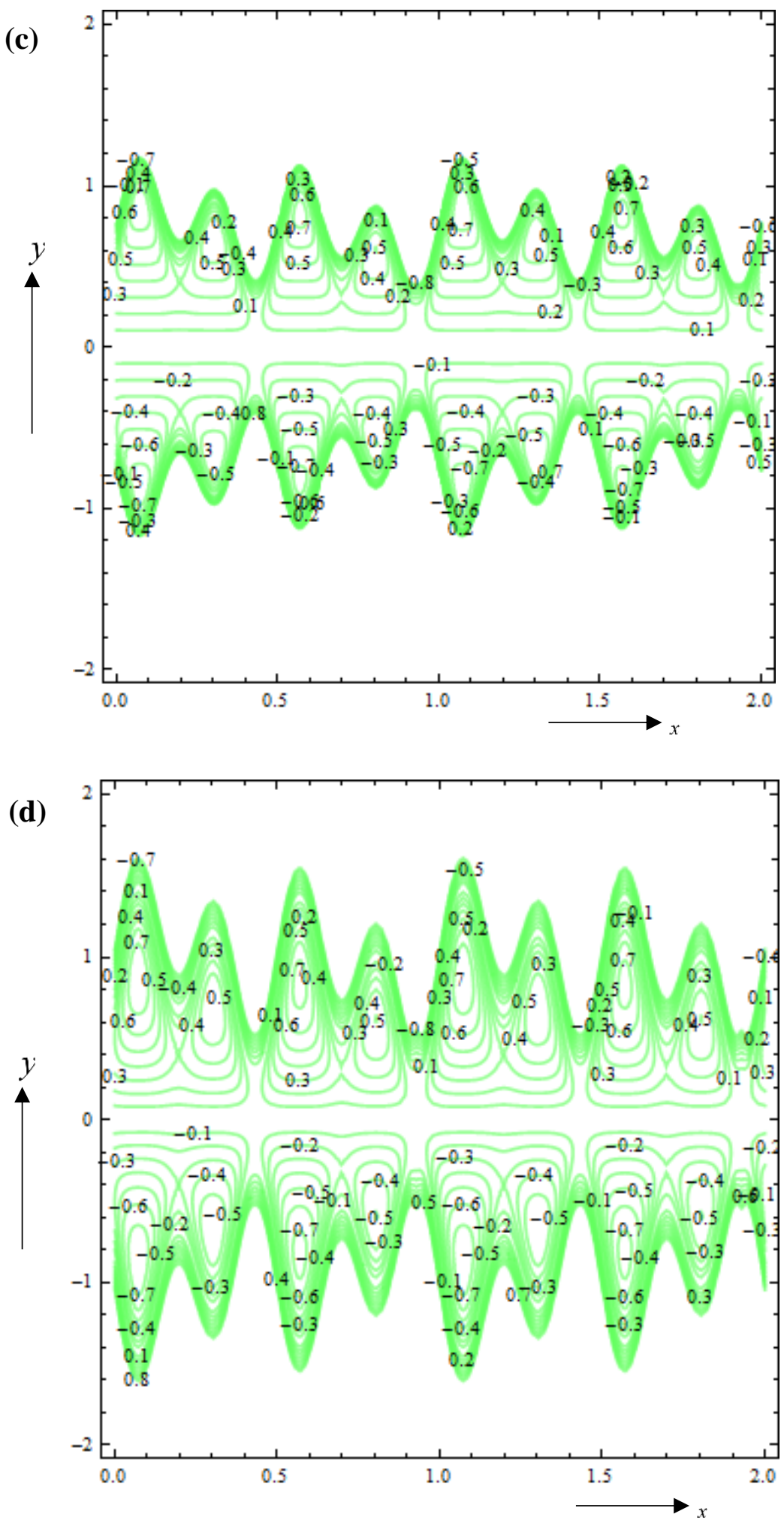


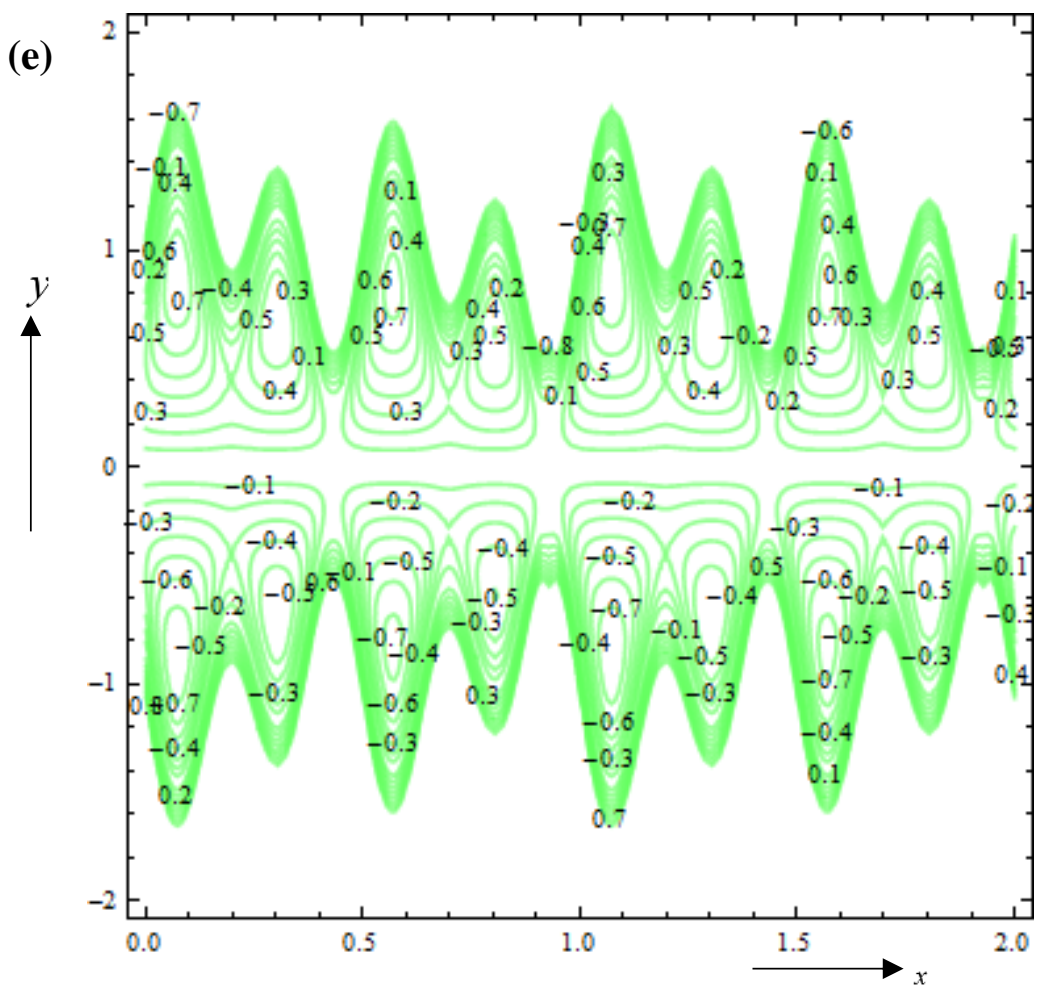

(f)

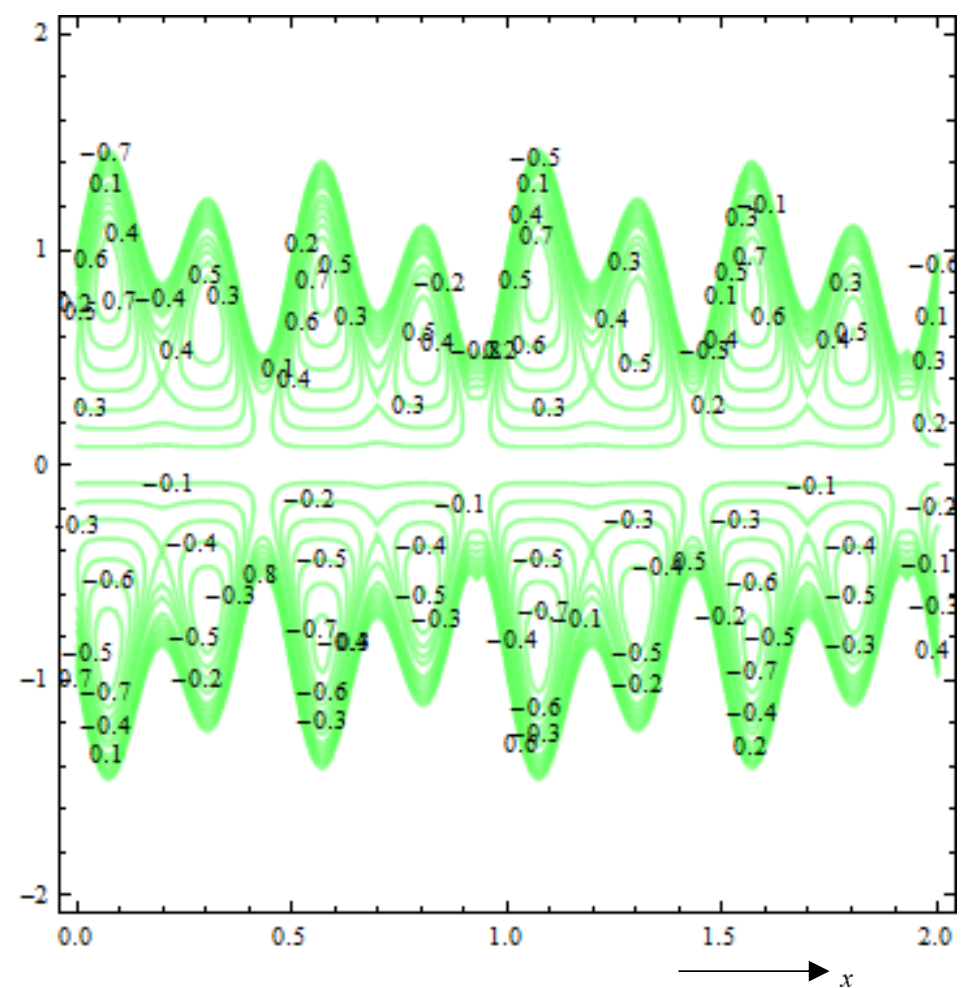




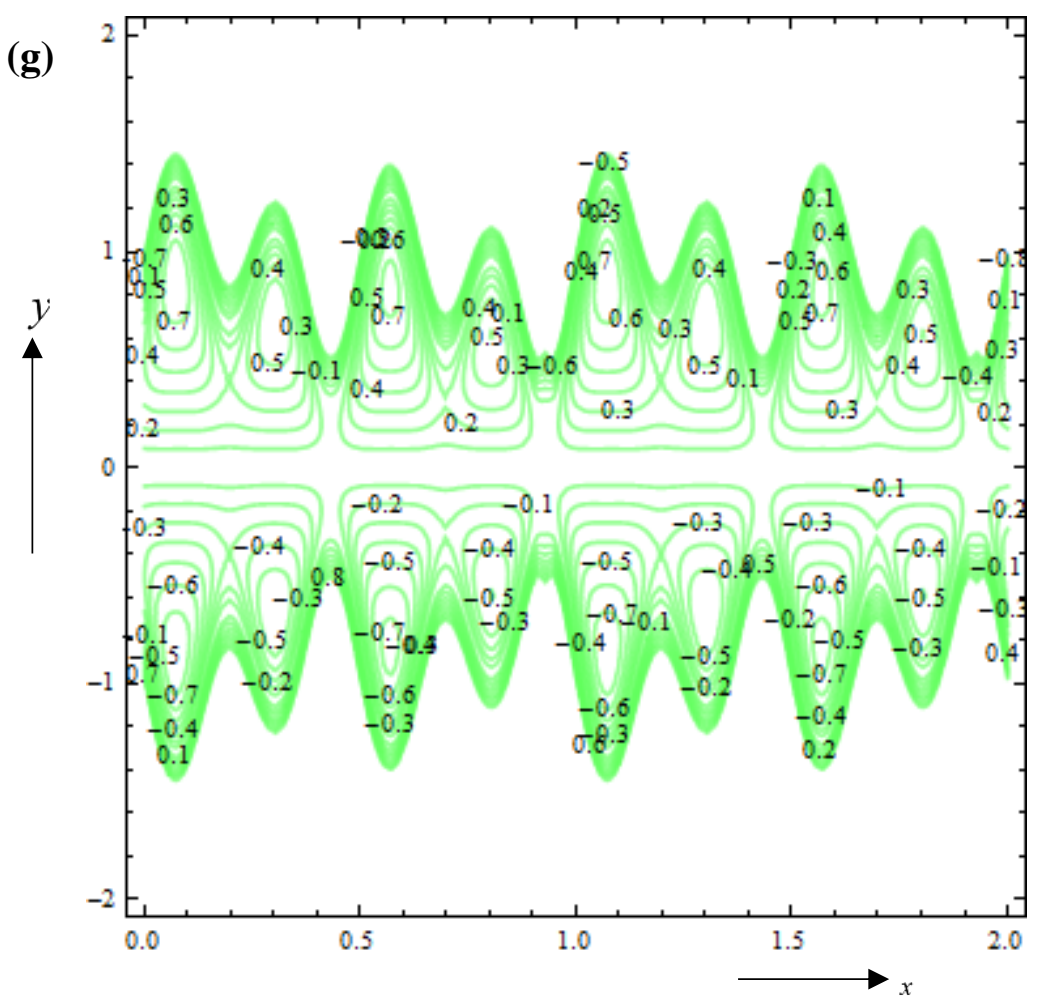

Fig.9. Contour plots for stream lines in wave form at $\varphi_{1}=0.1, \varphi_{2}=0.2, \varphi_{3}=0.3, \bar{Q}=0.9$ for (a) $m=1, \mathrm{U}_{H S}=1, k=0.1, \quad$ (b) $m=5, \mathrm{U}_{H S}=1, k=0.1, \quad$ (c) $m=10, \mathrm{U}_{H S}=1, k=0.1$, (d) $m=1, \mathrm{U}_{H S}=1, k=0.3$, (e) $m=1, \mathrm{U}_{H S}=1, k=0.5$, (f) $m=1, \mathrm{U}_{H S}=5, k=0.1$, (g) $m=1, \mathrm{U}_{H S}=10, k=0.1, \cdot$

Figs.3a-c illustrate the evolution of axial velocity $(u)$ with $m, U_{H S}$ and $k$, respectively. Increasing electro-osmotic parameter (which physically corresponds to decreasing Debye length or characteristic thickness of electrical double layer, EDL) is observed to initially strongly accelerate the axial flow.

However, as observed in fig.3a, for infinite values of $m$ there is a subsequent strong deceleration in the axial flow (greater negative values of axial velocity) especially near the channel walls. The profiles evolve from inverse parabolas about the channel centre line, to inverse plateauforms with an initial elevation in $m$ from finite to infinite values. The latter (infinite $m$ ) are associated with a vanishing Debye layer. Fig.3b reveals that with negative the HelmholtzSmoluchowski velocity $\left(U_{H S}\right)$, the axial velocity magnitudes are reduced whereas with a positive increase in $\left(U_{H S}\right)$, the axial flow is accelerated i.e. magnitudes are increased. Inverted parabolic profiles are however sustained for all values of the Helmholtz-Smoluchowski velocity $\left(U_{H S}\right)$ and the maximum magnitudes are computed around the core region i.e. channel centre line. With increasing permeability parameter $(k)$, as shown in fig. $3 \mathrm{c}$, there is a 
significant increase in the axial velocity magnitudes. The profiles are however alternating in nature with maximum values computed near the micro-channel walls. In the transformed axial momentum conservation eqn. (15), the Darcian porous media drag force term i.e. $-\mathrm{u} / \mathrm{k}$, is clearly inversely proportional to the permeability. As permeability increases, there is an associated depletion in solid fibers in the channel which results in a reduction in bulk drag from the porous matrix. This serves to accelerate the flow in the micro-channel. The permeability parameter $k$ is infact equivalent to a Darcy number based on the half width of the channel $(a)$. The values selected for $k$ represent very sparsely packed porous media i.e. high permeability regimes.

Figs. 4 a-b illustrate pressure difference $(\Delta p)$ versus axial distance for different time instants and different electro-osmotic parameters $(m)$. The multi-wave form is clearly captured in these plots which exhibit three different maxima and minima corresponding to the three distinct amplitudes of the three waves simulated in eqn. (1). The pressure distributions are therefore irregular. Increasing electro-osmotic parameter with finite values is found to initially elevate the pressure difference values at all axial locations i.e. for all values of $\xi$. However, with further increase in $m$ to infinite values, there is a noticeable reduction in pressure difference magnitudes. The peaks are displaced further along the axis i.e. further from the micro-channel entry, with progression in time i.e. as $t$ increases from 0.2 to 0.6 (figs. $4 \mathrm{a}$ and $4 \mathrm{~b}$ respectively).

Figs. 5a-b present the collective influence of time $(t)$ and Helmholtz-Smoluchowski velocity $\left(U_{H S}\right)$ on the axial evolution of pressure difference $(\Delta p)$. Increasing negative $U_{H S}$ is found to suppress pressure differences whereas increasing positive $U_{H S}$ is observed to accentuate pressure differences. The electro-osmotic effect therefore exerts a strong influence on pressure difference in the regime along the axis of the micro-channel. With elapse of time, again there is a migration in peak pressure differences further along the axial direction, although there is no tangible modification in magnitudes for any of the three different waves.

Figs. 6a-b present the effects of time $(t)$ and permeability parameter $(k)$ on pressure difference profiles with axial coordinate, $\xi$. Increasing permeability is observed to suppress pressure magnitudes whereas decreasing permeability increases them. This behaviour is sustained for all values of axial coordinate. The decrease in solid fibers present i.e. lower permeability assists in generating greater pressures in the regime. The selection of an appropriate permeability of the porous medium is therefore an important aspect controlling pressure distributions. With an increase in time, the distribution of pressure peaks for different waves is clearly modified with 
higher amplitude waves being displaced further along the axis and replaced with the smaller amplitude peaks.

Figs 7a-c depict the variation of initial flow rate with time for different hydrodynamic parameters. An increase in electro-osmotic parameter $(m)$ in fig. 7a clearly reduces flow rates since it is associated with a deceleration in the micro-channel flow. The three wave amplitudes are clearly captured in this and the other figures. Positive Helmholtz-Smoluchowski velocity $\left(U_{H S}\right)$ in fig. 7b clearly enhances initial flow rates (again due to associated flow acceleration in the regime) whereas negative Helmholtz-Smoluchowski velocity induces the opposite effect. The flow rate profiles are also much more dispersed than in fig. 7a where they are more clustered. Fig. 7c shows that with increasing permeability the magnitudes of the initial flow rate are increased, again due to acceleration of the flow in the micro-channel with a progressively more permeable matrix i.e. decreased Darcy impedance.

Figs 8a-c illustrate the evolution in local wall shear stress with axial coordinate, for different hydrodynamic parameters. An increase in electro-osmotic parameter $(m)$ in fig. 8a results in a marked depression in wall shear stress and again this is as a result of deceleration in the electrolyte flow in the micro-channel, especially at and near the wall zones. Positive Helmholtz-Smoluchowski velocity ( $\left.U_{H S}\right)$ manifests (as seen in fig. $7 \mathrm{~b}$ ) in an acceleration in the micro-channel flow near the walls and therefore elevates the local wall shear stress values. The converse effect is computed with negative value of Helmholtz-Smoluchowski velocity $\left(U_{H S}\right)$. The case of vanishing Helmholtz-Smoluchowski velocity $\left(U_{H S}=0\right)$ falls in between the other two cases. These results concur quite well with other published works including Maier et al. [11] and Li et al. [13]. With increasing permeability of the porous medium i.e. greater $k$ values, there is slight reduction in the shear stress magnitudes, as seen in fig. $8 \mathrm{c}$. In all the profiles the irregularity associated with three different wave amplitudes is clearly visualized.

Figs. 9a-g present the combined effects of the Helmholtz-Smoluchowski velocity $\left(U_{H S}\right)$, permeability parameter $(k)$ and electro-osmotic parameter $(m)$ on streamline distributions. In these plots, figs. 9a, 9b, 9c correspond to a change in electro-osmotic parameter from 1 to 5 to 10 , respectively. Although in the first two of these plots, very little modification in circulation and trapping patterns is observed, there is a change in strength of circulation zones in the third plot, in which bolus zones become more constricted and positive streamline magnitudes are altered to negative ones in the lower channel half space. Figs 9c,d,e show the respective streamline plots for a progressive increase in permeability parameter, $k$ from $0.1,0.3$ to 0.5 . 
There is a progressive expansion in the boluses and a relaxation in streamlines, associated with acceleration in the micro-channel as the permeability parameter is increased. A similar observation has been made by Obliger et al. [15]. The permeability of the porous medium therefore has a significant influence on the electro-kinetic fluid dynamics. Finally in figs. $9 \mathrm{f}, \mathrm{g}$ the Helmholtz-Smoluchowski velocity $\left(U_{H S}\right)$ is systematically increased from 5 to 10 with all other parameters held constant. No significant modification in bolus structure is observed and the distribution of the circulation zones remains generally unaltered.

\section{CONCLUSIONS}

A mathematical study has been conducted to investigate the electro-osmotic hydrodynamics in aqueous solution (electrolyte) pumping via a finite length micro-channel containing an isotropic porous medium. Three wave functions of different amplitudes but the same wavelength has been used to simulate a complex wavy surface for the micro-channel walls, corresponding to a more realistic peristaltic propulsion scenario. The Darcy model has been employed for linear porous media drag simulation. The transformed boundary value problem has been solved analytically. Validation with a finite difference method has been included. The present analysis has shown that higher values of electro-osmotic parameter i.e. lower values of electrical Debye length decrease flow rates and decelerate the micro-channel flow. An increase in matrix permeability is observed to suppress pressure difference magnitudes for all three peristaltic waves. Negative Helmholtz-Smoluchowski velocity suppresses pressure differences whereas increasing positive values generate an increase in pressure differences. With progress in time, peak pressure differences are displaced along the microchannel axis i.e. the peaks are spaced differently along the channel length for the three different waves considered. With negative Helmholtz-Smoluchowski velocity $\left(U_{H S}\right)$, axial flow is decelerated. Axial flow acceleration is also observed with increasing permeability parameter. Trapping phenomena are also studied and permeability is observed to have a strong influence on circulation and bolus structure. The present work considered axial electrical field effects. Future studies will also consider transverse magnetic fields [38] and efforts in this regard are currently underway.

\section{REFERENCES}

1. N. Kasayapanand and T. Kiatsiriroat, EHD enhanced heat transfer in wavy channel, Int. Comm. Heat Mass Transfer, 32, 809-821 (2005). 
2. C-O. Ng and Q. Zhou, Electro-osmotic flow through a thin channel with gradually varying wall potential and hydrodynamic slippage, Fluid Dyn. Res. 44055507 (2012).

3. A. Marcos, Y. J. Kang, K. T. Ooi, C. Yang and T. N. Wong, Frequency-dependent velocity and vorticity fields of electro-osmotic flow in a closed-end cylindrical microchannel, J. Micromechanics and Microengineering, 15301 (2004).

4. G. D. Ngoma and F. Erchiqui, Pressure gradient and electroosmotic effects on two immiscible fluids in a microchannel between two parallel plates, J. Micromech. Microeng. 1683 (2005).

5. K. Kim, H. S. Kwak and T-H. Song, A numerical model for simulating electroosmotic micro- and nanochannel flows under non-Boltzmann equilibrium, Res. 43041401 (2011).

6. K.-D. Huang and R-J. Yang, Electrokinetic behaviour of overlapped electric double layers in nanofluidic channels, Nanotechnology 18115701 (2007).

7. Y. Ren and D. Stein, Slip-enhanced electrokinetic energy conversion in nanofluidic channels, Nanotechnology, 19, 195707 (2008).

8. W. Zhang et al., Analysis of electrokinetic coupling of fluid flow in porous media using a 3-D pore network, J. Petroleum Science and Engineering, 134,_150-157 (2015).

9. R. Azzam and W. Oey, The utilization of electrokinetics in geotechnical and environmental engineering, Transport in Porous Media, 42, 293-314 (2001).

10. D. B. Pengra et al., Experimental study of electrokinetics in porous media, MRS Proceedings,366, 201 (1994).

11. R.S. Maier et al., Electroosmotic flow and dispersion in open and closed porous media, Transport in Porous Media, 113, 67-89 (2016).

12. A. Gupta, D. Coelho and P.M. Adler, Universal electro-osmosis formulae for porous media, J. Colloid Interface Science, 319, 549-554 (2008).

13. B. Li, W.N. Zhou, Y.Y. Yan and C. Tian, Evaluation of electro-osmotic pumping effect on microporous media flow, Applied Thermal Engineering, 60, 449-455 (2013).

14. A.K. Gupta, D. Coelho and P.M. Adler, Influence of the Stern layer on electro-kinetic phenomena in porous media, J. Colloid Interface Science, 316, 140-159 (2007).

15. A. Obliger, M. Jardat, D. Coelho, S. Bekri and B. Rotenberg, Pore network model of electrokinetic transport through charged porous media, Phys. Rev. E, 89, 043013 (2014).

16. S. Di Fraia, N. Massarotti and P. Nithiarasu, Finite element modelling of electroosmotic flow in porous media, Proc. 24th UK Conference of the Association for 
Computational Mechanics in Engineering, 31 March - 01 April, Cardiff University, Cardiff(2016).

17. G.H. Tang, P.X. Ye, W.Q. Tao, Pressure-driven and electroosmotic non-Newtonian flows through microporous media via lattice Boltzmann method, J. Non-Newtonian Fluid Mech. 165, 1536-1542 (2010).

18. M. She and Z. Liu, CFD simulation and experimental study of electroosmotic flow in porous media. (I). CFD simulation of electroosmotic flow in porous media, J. Chemical Industry and Engineering (China) 54(8):1037-1043 (2003).

19. Z. Brož and N. Epstein, Electrokinetic flow through porous media composed of fine cylindrical capillaries, J. Colloid and Interface Science, 56, 605-612 (1976).

20. Y. Y. Wu and H. J. Keh, Electrokinetic flow and electric current in a fibrous porous medium, J. Phys. Chem. B, 116 (11), 3578-3586 (2012).

21. C. Yang, Y. Kang, X. Huang, Electrokinetic flow in porous media, Encyclopedia of Microfluidics and Nanofluidics,_506-516 (2008).

22. Z. Chai, Z. Guo and B. Shi, Study of electro-osmotic flows in microchannels packed with variable porosity media via lattice Boltzmann method, J. Appl. Phys., 101, 104913 (2007).

23. D. Piwowar, Characterization and Performance of the Electroosmotic Pumping Effect for Different Porous Media, MSc, Rutgers University, Mechanical and Aerospace Engineering (2012).

24. E.F. El Shehawey, A.M.F. Sobh, E.M.E. Elbarbary, Peristaltic motion of a generalized Newtonian fluid through a porous medium, Phys. Soc. Japan, 69: 401-407 (2000).

25. T. Hayat, N. Ali, S. Asghar, Hall effects on peristaltic flow of a Maxwell fluid in a porous medium, Physics Letters A., 363: 397- 403 (2007).

26. K. Vajravelu, G. Radhakrishnamacharya and V. Radhakrishnamurthy, Peristaltic flow and heat transfer in a vertical porous annulus, with long wave approximation, Int. J. Non-Linear Mech., 42, $754-759$ (2007).

27. C. Vasudev, U. Rajeswara Rao, M.V. Subba Reddy and G. Prabhakara Rao, Effect of heat transfer on peristaltic transport of a Newtonian fluid through a porous medium in an asymmetric vertical channel, European J. Scientific Research, 44, 79 - 92 (2010).

28. D. Tripathi, O.A. Bég, A numerical study of oscillating peristaltic flow of generalized Maxwell viscoelastic fluids through a porous medium, Transport in Porous Media, 95 (2), 337-348 (2012). 
29. O.A. Bég, T.A. Bég, R. Bhargava, S. Rawat and D. Tripathi, Finite element study of transient pulsatile magneto-hemodynamic non-Newtonian flow and drug diffusion in a porous medium channel, J. Mechanics Medicine/Biology, 12 (04), 1250081 (2012).

30. C.C. Cho and C.L. Chen, Characteristics of transient electroosmotic flow in microchannels with complex-wavy surface and periodic time-varying electric field, ASME J. Fluids Engineering, 135(2), 021301 (2013).

31. N. Al Quddus, S. Bhattacharjee and W. Moussa, Electrokinetic flow in a wavy channel, Int. Conf. MEMS, NANO/Smart Systems (ICMENS'05), Washington (2005).

32. N. Quddus, S. Bhattacharjee, and W. Moussa, An electrostatic-peristaltic colloidal micropump: a finite elemental analysis, J. Comp. Theor. Nanoscience, 1, 1-7 (2005).

33. J. Bear, Dynamics of Fluids in Porous Media, Dover, New York (1972).

34. H.B. Keller, A new difference method for parabolic problems, J. Bramble (Editor), Numerical Methods for Partial Differential Equations, Academic Press, USA (1970).

35. M.Y. Malik, T. Salahuddin, A. Hussain, and S. Bilal, MHD flow of tangent hyperbolic fluid over a stretching cylinder using Keller box method, J. Magnetism Magnetic Materials, 395 (2015) 271-276.

36. O. Anwar Bég, S. Abdul Gaffar, V. R. Prasad and M.J. Uddin, Computational solutions for non-isothermal, nonlinear magnetoconvection in porous media with Hall/ionslip currents and Ohmic dissipation, Eng. Science Tech., 18 pages (2015). DOI.ORG/10.1016/ J.JESTCH.2015.08.009

37. A. Subba Rao, V.R. Prasad, K. Harshavalli and O. Anwar Bég, Thermal radiation effects on non-Newtonian fluid in a variable porosity regime with partial slip, J. Porous Media, 19 (2016) 1-17.

38. D. Tripathi, S. Bhushan and O. Anwar Bég, Transverse magnetic field driven modification in unsteady peristaltic transport with electrical double layer effects, Colloids and Surfaces A: Physicochemical and Engineering Aspects, 506, 32-39 (2016). 